\title{
DERIVATION OF THREE CLOSED LOOP KINEMATIC VELOCITY MODELS USING NORMALIZED QUATERNION FEEDBACK FOR AN AUTONOMOUS REDUNDANT MANIPULATOR WITH APPLICATION TO INVERSE KINEMATICS
}

\author{
M. A. Unseren
}

DATE PUBLISHED - April 1993

Research sponsored by the Engineering Research Program Office of Basic Energy Sciences and by the

Office of Technology Support Programs

Office of Nuclear Energy

U.S. Department of Energy

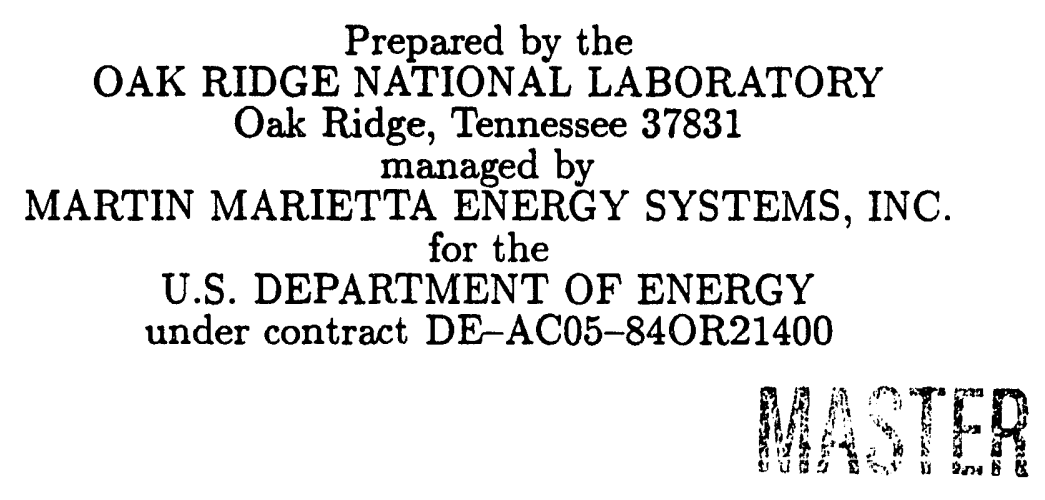




\section{CONTENTS}

ABSTRACT . . . . . . . . . . . . . . . . v

1. INTRODUCTION AND MOTIVATION . . . . . . . . . . . . . 1

2. PROBLEM STATEMENT AND SYSTEM DESCRIPTION . . . . . . . 7

2.1 SYSTEM VARIABLES AND COORDINATE FRAMES . . . . . . 7

2.2 KINEMATIC TRANSFORMATIONS . . . . . . . . . . . . . . 7

3. REVIEW OF QUATERNION ALGEGRA . . . . . . . . . . . . . 9

3.1 DEFINITION OF THE QUATERNION . . . . . . . . . . . . . . . 9

3.2 GENERAL PROPERTIES OF NORMALIZED QUATERNION ALGEBRA . . . . . . . . . . . . . . . . . . . . 11

3.3 SUCCESSIVE ROTATIONS AND REPRESENTATIONS WITH RESPECT TO VARIOUS COORDINATE SYSTEMS . . . . . . . 12

4. ORIENTATION ERROR ANALYSES . . . . . . . . . . . . . . . . 15

4.1 APPLICATION OF ICKES ORIENTATION ERROR TO REDUNDANT MANIPULATORS . . . . . . . . . . . . . . . 15

4.2 APPLICATION OF LWP ORIENTATION ERROR TO REDUNDANT MANIPULATORS . . . . . . . . . . . . . . . . 17

4.3 A NEW ERROR METHOD WITH APPLICATION TO REDUNDANT MANIPULATORS . . . . . . . . . . . . . . . 21

5. REDUNDANCY RESOLUTION . . . . . . . . . . . . . . . 25

5.1 INPUT RELEGATION CONTROL METHOD . . . . . . . . . 25

5.2 TWO NON-REDUNDANT STEP METHOD . . . . . . . . . . . 27

6. CONCLUSION AND FUTURE WORK . . . . . . . . . . . . . . 29

7. ACKNOWLEDGMENT . . . . . . . . . . . . . . . . . 31 APPENDIX A: SINGULARITIES IN COMPUTING EULER ANGLES, THEIR TIME DERIVATIVES, AND JACOBIAN $J^{*}(q) \ldots . . . . .33$ APPENDIX B: DERIVATION OF ICKES ORIENTATION ERROR . . . . . 37 APPENDIX C: QUATERNION PRODUCT SIMPLIFICATIONS . . . . . . 39 APPENDIX D: CLOSED LOOP TRANSLATIONAL ERROR SUBSYSTEM . . . . . . . . . . . . . . . . . 41

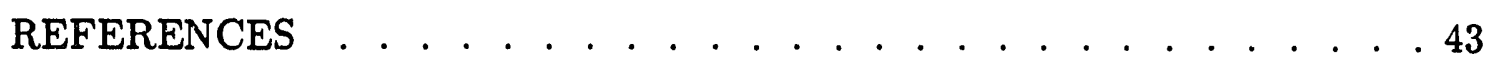




\section{LIST OF FIGURES}

Fig.

Page

1 System configuration and coordinate system assignment . . . . . . 4

2 Rotation of $\left(X_{0}, Y_{0}, Z_{0}\right)$ coordinate frame about unit vector $\vec{n}$ by angle $\theta$ to obtain $\left(X_{N}, Y_{N}, Z_{N}\right)$ coordinate frame . . . . . 10

3 Open loop redundancy resolution to the plant . . . . . . . . . 28

4 Closed loop redundancy resolution to the plant . . . . . . . . . 28 


\begin{abstract}
The report discusses the orientation tracking control problem for a kinematically redundant, autonomous manipulator moving in a three dimensional workspace. The orientation error is derived using the normalized quaternion error method of Ickes [7], the Luh, Walker, and Paul error method [11], and a method suggested here utilizing the Rodrigues parameters, all of which are expressed in terms of normalized quaternions. The analytical time derivatives of the orientation errors are determined. The latter, along with the translational velocity error, form a closed loop kinematic velocity model of the manipulator using normalized quaternion and translational position feedback. An analysis of the singularities associated with expressing the models in a form suitable for solving the inverse kinematics problem is given. Two redundancy resolution algorithms originally developed using an open loop kinematic velocity model of the manipulator are extended to properly take into account the orientation tracking control problem. This report furnishes the necessary mathematical framework required prior to experimental implementation of the orientation tracking control schemes on the seven axis CESARm research manipulator $[21,36,37,38]$ or on the seven-axis Robotics Research K1207i dexterous manipulator, the latter of which is to be delivered to the Oak Ridge National Laboratory in 1993.
\end{abstract}




\section{INTRODUCTION AND MOTIVATION}

The basic theory on the parameterization of orientation (attitude) of a rigid body in a three-dimensional workspace is well established $[1,2,3,4,5]$. The generalized coordinates parameterizing orientation include the $\mathrm{XYZ}, \mathrm{ZYZ}$, and $\mathrm{ZXZ}$ convention Euler angles [1] (the XYZ convention is termed roll, pitch, and yaw angles by many authors), orthogonal rotation matrix (i.e., di. oction cosine matrix) [1], Euler rotation angle and unit vector [1,2], Cayley-Klein parameters [1], normalized quaternions (i.e., Euler Parameters) $[1,2,3,4,5,6]$, and Rodrigues parameters [2, 5]. The application of this theory to the problem of controlling the orientation of a rigid body to track a desired, reference trajectory in a three-dimensional workspace requires the determination and mathematical modeling of the error between the desired and actual orientations of the rigid body. This problem is complicated by the fact that the Cartesian angular velocity vector, which is often used to describe the rotational motion of a spatial rigid body [6], is not the time derivative of a vector [1] (whereas Cartesian translational velocity is the direct time derivative of translational position). Indeed, angular velocity is referred to as a nonholonomic vector in [1]. One of the earliest researchers to address this problem was Ickes [7]. It was argued in [7] that the desired and actual normalized quaternions are an appropriate set of generalized coordinates to form the orientation error needed for control purposes. A mathematical model of the orientation error was derived in [7] which has served as the basis for recent work on this problem in robotics $[8,9,10]$. Another approach to formulating the error between the desired and actual end effector orientations using orthogonal rotation matrices or the Euler rotation angle and unit vector is suggested in [11]. It is termed the Luh, Walker, Pau! (LWP) orientation error. The previous work on the orientation error control problem in autonomous robotics has mainly been restricted to the resolved rate and resolved acceleration control of kinematically nonredundant manipulators.

On the other hand, a critical view of the literature on the theory, modeling, and control of kinerratically redundant autonomous manipulators $[12,13,14,15,16,17$, $18,19,20]$ reveals that the spatial orientation control problem has largely been ignored. These literature resolve the redundancy based on open loop kinematic velocity and/or acceleration models of a serial link manipulator which could conceivably be applied to a spatial arm with $N(\geq 7)$ joints. But the proof of principle simulation examples presented in these literature mostly focus on the inverse kinematics of redundant planar manipulators with revolute joints or as in [17], a 3 DOF planar manipulator with two prismatic and one revolute joint. But the orientation of a planar rigid body, e.g., the end effector of a planar revolute serial link manipulator, can be represented by a single Euler angle measured about an axis perpendicular to the plane of motion. Only the component of angular velocity measured about the axis perpendicular to the plane is nonzero and it is simply the time derivative of the Euler 
angle measured about that axis. Thus even if feedback loops are incorporated nito the model for servoing of the tracking errors, the orientation error can be formed in the same way that the translational position error is formed in such a configuration. Thus the spatial orientation problem has been avoided. In [21, 22], the LWP orientation error was formed by employing orthogonal rotation matrix feedback [11] for the resolution of redundancy of 7 DOF and 9 DOF spatial manipulators, respectively. But only three of the nine parameters of a rotation matrix are independent, and the representation of the LWP error in terms of the four parameter normalized quaternions will be investigated here. Besides, normalized quaternions are computationally efficient $[1,23]$. The time derivative of the LWP error, which was not considered in $[11,21,22]$, will be discussed in this report.

Some comments are in order about the $(6 \times N)$ Jacobian matrix $J^{*}(q)$ that is contained in the open loop kinematic velocity model upon which several researchers base redundancy resolution schemes $[12,13,14,15]$. The Jacobian and model are derived by the following procedure in these literature: Let $X$ denote a $(6 \times 1)$ task or operational space vector. The upper three elements of $X$ are the components of Cartesian translational position of the end effector at its centerpoint. The lower three elements of $X$ are not specifically identified, but are assumed to be a set of Euler angles $[\alpha, \beta, \gamma]^{T}$ describing the orientation of the end effector, where superscript $T$ denotes a matrix transposition. The task vector $X$ is expressed as a function of the joint positions $q\left(=\left[q_{1}, q_{2}, \ldots, q_{N}\right]^{T}\right)(N \geq 7)$ :

$$
X=H
$$

where the $(6 \times 1)$ vector $H(q)$ is a continuous function of its argument. The Jacobian $J^{*}$ is defined to be the $(6 \times N)$ matrix $(\partial H / \partial q)$ in the equation obtained by differentiating eq. (1) :

$$
\dot{X}=(\partial H / \partial q) \dot{q}=J^{*} \dot{q} .
$$

It should be noticed that the lower three elements of $\dot{X}$ are merely the time rates of change of the Euler angles. Indeed, differentiating the lower three elements of $X$, regardless of whatever 3-element parameterization of orientation they may be, will never yield components of Cartesian angular velocity of the end effector, since, as mentioned earlier, angular velocity is not the time derivative of a vector. Only the upper three rows of $J^{*}$ as defined in eq. (2) constitute a joint to Cartesian space mapping. The lower three rows of eq. (2) are in fact a mapping from one set of angular coordinates to another. To derive the lower three rows of $J^{*}$ symbolically using the above procedure, the designer must first obtain analytical expressions for the lower three elements of $H$ to satisfy the equation $[\alpha, \beta, \gamma]^{T}=\left[0_{3 \times 3}, I_{3 \times 3}\right] H(q)$. ( Here $I_{r \times r}$ denotes an $(r \times r)$ identity matrix and $0_{r \times s}$ an $(r \times s)$ matrix of zeros ). To express Euler angles as functions of the joint positions involves extracting the former from a $(3 \times 3)$ orthogonal rotation matrix ${ }^{a} R_{0}^{N}(q)$ which describes the actual orientation of the 
end effector moving coordinate system $\left(X_{N}, Y_{N}, Z_{N}\right)$ with respect to the stationary base coordinate frame $\left(X_{0}, Y_{0}, Z_{0}\right)[6]$. The coordinate frames are shown in Fig. 1. However, this procedure involves the use of the two argument arctangent function (due to the nonuniqueness of Euler angles) and degeneracies cannot be avoided [6,24]. The inherent degeneracy of the extraction algorithm suggested in [6] is demonstrated for the XYZ, ZYZ, and ZXZ Euler angle conventions in Appendix A. By simulating planar redundant manipulators in their examples, the literature $[12,13,14,15]$ which define $J^{*}$ by eqs. (1) and (2) have in fact avoided this problem.

On the other hand, the Jacobian matrix $J(q)$ presented in this report transforms the joint velocities to obtain the Cartesian t. nslational and angular velocities of the end effector in the base coordinates ( $J$ is defined mathematically in the next section). The lower three rows of this Jacobian can be derived symbolically using a well established algorithm described in $[25,26]$ which has no degeneracies.

There is a second method for determining vector $[\dot{\alpha}, \dot{\beta}, \dot{\gamma}]^{T}$ as a function of the joint velocities. It involves two steps: (i) calculate the Cartesian angular velocity of the end effector given the lower three rows of $J$ and $\dot{q}$. (ii) calculate $[\dot{\alpha}, \dot{\beta}, \dot{\gamma}]^{T}$ as a function of the angular velocity and $[\alpha, \beta, \gamma]^{T}$. In Appendix $\mathrm{A}$ it is shown for the $\mathrm{XYZ}, \mathrm{ZYZ}$, and ZXZ conventions that step (ii) has singularities and that they are identical to the singularities found when extracting $[\alpha, \beta, \gamma]^{T}$ from ${ }^{a} R_{0}^{N}$. The procedure described in steps (i) and (ii) results in the determination of $J^{*}$ as a function of $J$ and $[\alpha, \beta, \gamma]^{T}$ [10], and in Appendix A it is shown that the singularities of this function are identical to those in step (ii). It is evident that modeling the manipulator system using eq. (2) and introducing feedback loops to control $[\dot{\alpha}, \dot{\beta}, \dot{\gamma}]^{T}$ to track a desired trajectory is impractical and another representation of orientation should be considered.

The spatial orientation error methods presented in this report employ normalized quaternion feedback. There are two significant advantages for using the normalized quaternions over Euler angles. Firstly, there exists an algorithm [27] for extracting the normalized quaternions from an orthogonal rotation matrix ${ }^{a} R_{0}^{N}$ which is singularity free except for the case when the Euler rotation angle $\theta$, which is defined in Section 3 , equals $\pm 180^{\circ}$. It is the opinion of the author that the singularities associated with extracting normalized quaternions from ${ }^{a} R_{0}^{N}$ are less restrictive than those arising from extracting Euler angles. Secondly, and most important, is the fact that the time derivatives of the normalized quaternions can be expressed as functions of the Cartesian angular velocity and normalized quaternions which are singularity free. It should be mentioned that the expressions for the orientation error derived here are Cartesian space vectors.

In this report the existing orientation error methods of $[7,11]$ and a method proposed here using the Rodrigues parameters are applied to the problem of deriving a closed loop kinematic velocity model of an autonomous redundant manipulator. The method of [7] is based on a four parameter representation of orientation whereas 


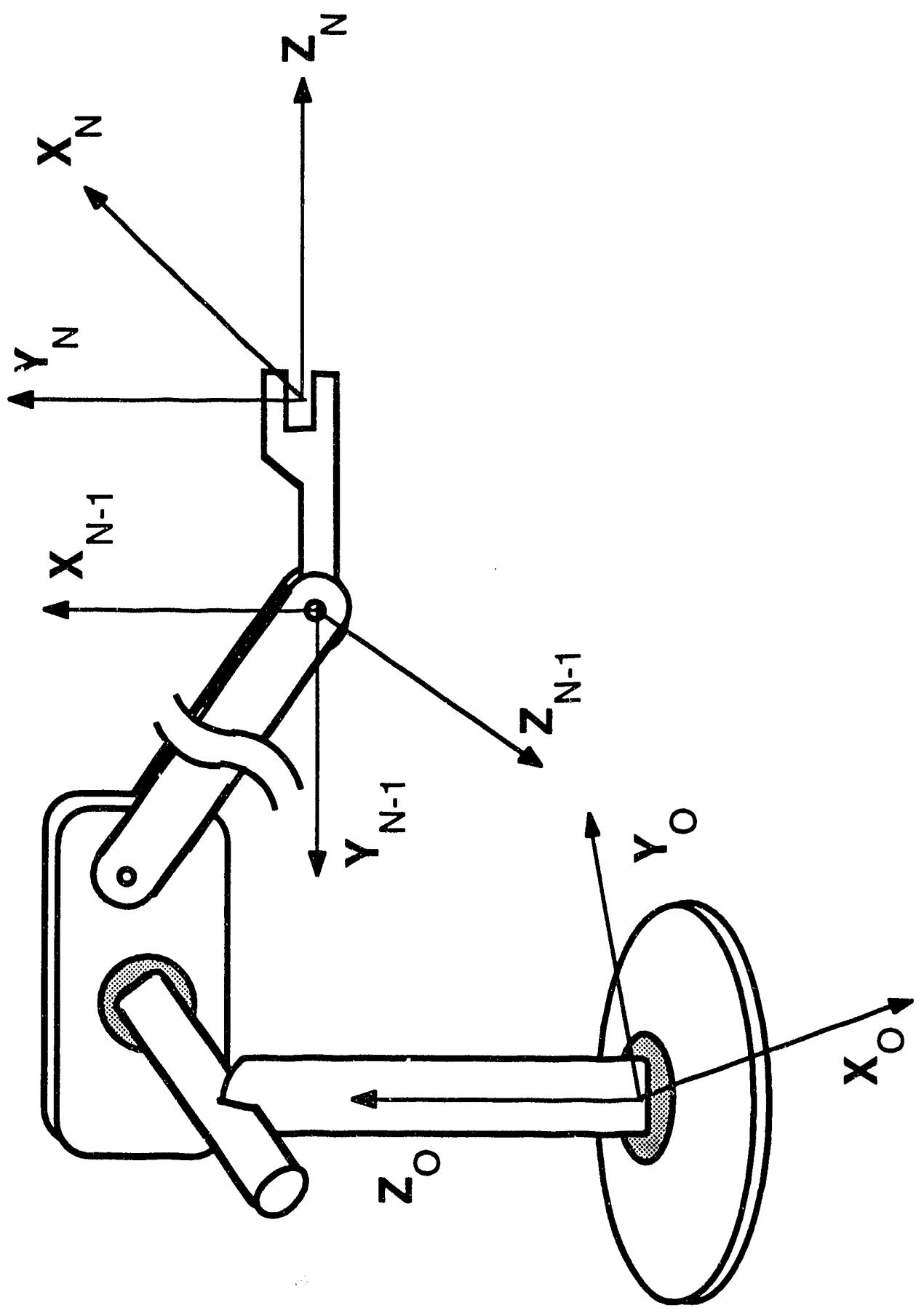

范 
the method of [11] is based on nine parameters (orthogonal rotation matrix) or four parameters (Euler rotation angle and unit vector). The proposed method is based on a three parameter representation of orientation. An analytical expression for the time derivative of each of three orientation errors is derived and combined with the translational velocity error, to yield a distinct closed loop kinematic velocity model for the entire system. The singularities of the error systems for the rotational kinematics are determined. The models form the basis for theoretical and experirnental comparisons of the three orientation error control methods. Two redundancy resolution algorithms originally developed based on an open loop kinematic velocity model of a manipulator are extended to solve for the joint velocities using the models given here to properly take into account the spatial orientation tracking control problem.

The report is organized as follows. First, the algebra of normalized quaternions relevant to this report is briefly reviewed. The orientation error, its analytical time derivative, and a closed loop system model at the velocity level for each of the three methods is presented. The problem of resolving the kinematic redundancy based on the models is then studied. Finally, a conclusion and recommendation for future work are provided. 


\section{PROBLEM STATEMENT AND SYSTEM DESCRIPTION}

The problem is to determine a closed loop kinematic velocity model for an autonomous, serial-link, redundant manipulator which takes into account the spatial orientation tracking control problem. Three such models are derived here, each of which is derived by a distinct orientation error method. The models serve as a proper foundation for the resolution of kinematic redundancy. The manipulator has a stationary base and contains $N(\geq 7)$ single DOF joints. The configuration of the system is shown in rig. 1 .

\subsection{SYSTEM VARIABLES AND COORDINATE FRAMES}

The joint positions $q=\left[q_{1}, q_{2}, \ldots, q_{N}\right]^{T}$ of the manipulator are the generalized coordinates describing the configuration of the system. The system variables include the generalized coordinates and velocities, the Cartesian velocities of the rigid body end effector, and the normalized quaternions. As shown in Fig. 1, the coordinate frame $\left(X_{k}, Y_{k}, Z_{k}\right)$ is assigned to the $k_{t h}$ link of the manipulator, where $k=0,1, \ldots, N$.

Let $p_{0}^{N}$ denote the $(3 \times 1)$ translation vector which emanates from the origin of the $\left(X_{0}, Y_{0}, Z_{0}\right)$ coordinate frame to the origin of the $\left(X_{N}, Y_{N}, Z_{N}\right)$ coordinate frame. $p_{0}^{N}$ is expressed in the base coordin ites.

\subsection{KINEMATIC TRANSFORMATIONS}

The kinematic transformation for a serial-link redundant manipulator which relates the Cartesian velocities of the end effector to the generalized velocities is given by:

$$
\left[\begin{array}{c}
v_{0}^{N} \\
\omega_{0}^{N}
\end{array}\right]=J \dot{q}
$$

where the $(3 \times 1)$ vectors $v_{0}^{N}\left(=\dot{p}_{0}^{N}\right)$ and $\omega_{0}^{N}$ are the Cartesian translational and angular velocities of the end effector in the base coordinates. In eq. $(3), J(q)$ is the $(6 \times N)$ manipulator Jacobian matrix, which is assumed to possess full rank six. It is convenient to partition $J$ into two matrices:

$$
J=\left[\begin{array}{l}
J_{t} \\
J_{r}
\end{array}\right]
$$

where $J_{t}(q)$ and $J_{r}(q)$ are $(3 \times N)$ matrices which transform $\dot{q}$ to obtain the translational and angular velocities of the end effector, respectively.

In this report eq. (3) is viewed only as a kinematic transformation for expressing the actual values of the end effector Cartesian velocities, denoted by $\left\{{ }^{a} v_{0}^{N},{ }^{a} \omega_{0}^{N}\right\}$, as 
symbolic functions of the joint space variables $\{q, \dot{q}\}$. It is not viewed as a model for solving the inverse kinematics, i.e., $\left\{{ }^{d} v_{0}^{N},{ }^{d} \omega_{0}^{N}, q\right\}$ are given and the problem is to determine an underspecified solution for $\dot{q}$, where superscript $d$ denotes the desired, planned values of the end effector Cartesian velocities.

A brief review of the fundamentals of quaternion algebra relevant to this report is covered in the next section. 


\section{REVIEW OF QUATERNION ALGEBRA}

According to Euler's Theorem on Rigid Body Rotations [1], the orientation (attitude) of a rigid body, after having undergone a sequence of rotations, is equivalent to a single rotation of that rigid body about an axis (unit vector) $\vec{n}$ by an angle $\theta$. The superscript $\rightarrow$ denotes that the quantity is an explicit function of the unit vectors directed along the principal axes of the Cartesian coordinate system it is expressed in. $\vec{n}$ and $\theta$ are shown in Fig. 2, where for illustrative purposes, the origins of the base and end effector coordinate systems coincide. The components of $\vec{n}$, denoted by a $(3 \times 1)$ vector $n=\left[n_{x}, n_{y}, n_{z}\right]^{T}$, may be expressed in any relevant Cartesian coordinate system, but will be in terms of the $\left(X_{0}, Y_{0}, Z_{0}\right)$ coordinate system in this report. Since all quantities and results are presented in a matrix/column vector notation throughout the report, vector $n$ is used hereinafter. In the quaternion description of the orientation of a rigid body, a $(4 \times 1)$ vector $e$ is introduced, termed the quaternion. It consists of a scalar component $e_{s}$ and a (Cartesian) vector component $e_{v}\left(=\left[e_{v x}, e_{v y}, e_{v z}\right]^{T}\right)$ :

$$
e=\left[\begin{array}{l}
e_{s} \\
e_{v}
\end{array}\right]
$$

\subsection{DEFINITION OF THE QUATERNION}

The quaternion $e$ can be defined in terms of $(n, \theta)[1]$ :

$$
e(n, \theta)=\left[\begin{array}{r}
\cos (\theta / 2) \\
n \sin (\theta / 2)
\end{array}\right] \text {. }
$$

$e_{v}$ contains the components of $\vec{e}_{v}$, and eq. (6) reveals that $\vec{e}_{v}$ is in alignment with $\vec{n}$ as illustrated in Fig. 2. It is immediately evident that the square of the Euclidean norm of $e$ equates to unity:

$$
\left(\|e\|_{2}\right)^{2}=e_{s}^{2}+e_{v x}^{2}+e_{v y}^{2}+e_{v z}^{2}=1 .
$$

Thus, $e$ is termed a normalized quaternion and its components are referred to as Euler Parameters [1]. Interestingly, the constraint equation (7) shows that the absolute value of any Euler Parameter cannot exceed unity. It is easy to see that:

$$
e^{T} \dot{e}=0
$$

Since the following relation holds:

$$
e(n, \theta)=\left[\begin{array}{cc}
1 & 0_{1 \times 3} \\
0_{3 \times 1} & -I_{3 \times 3}
\end{array}\right] e(n,-\theta)=e(-n,-\theta)
$$




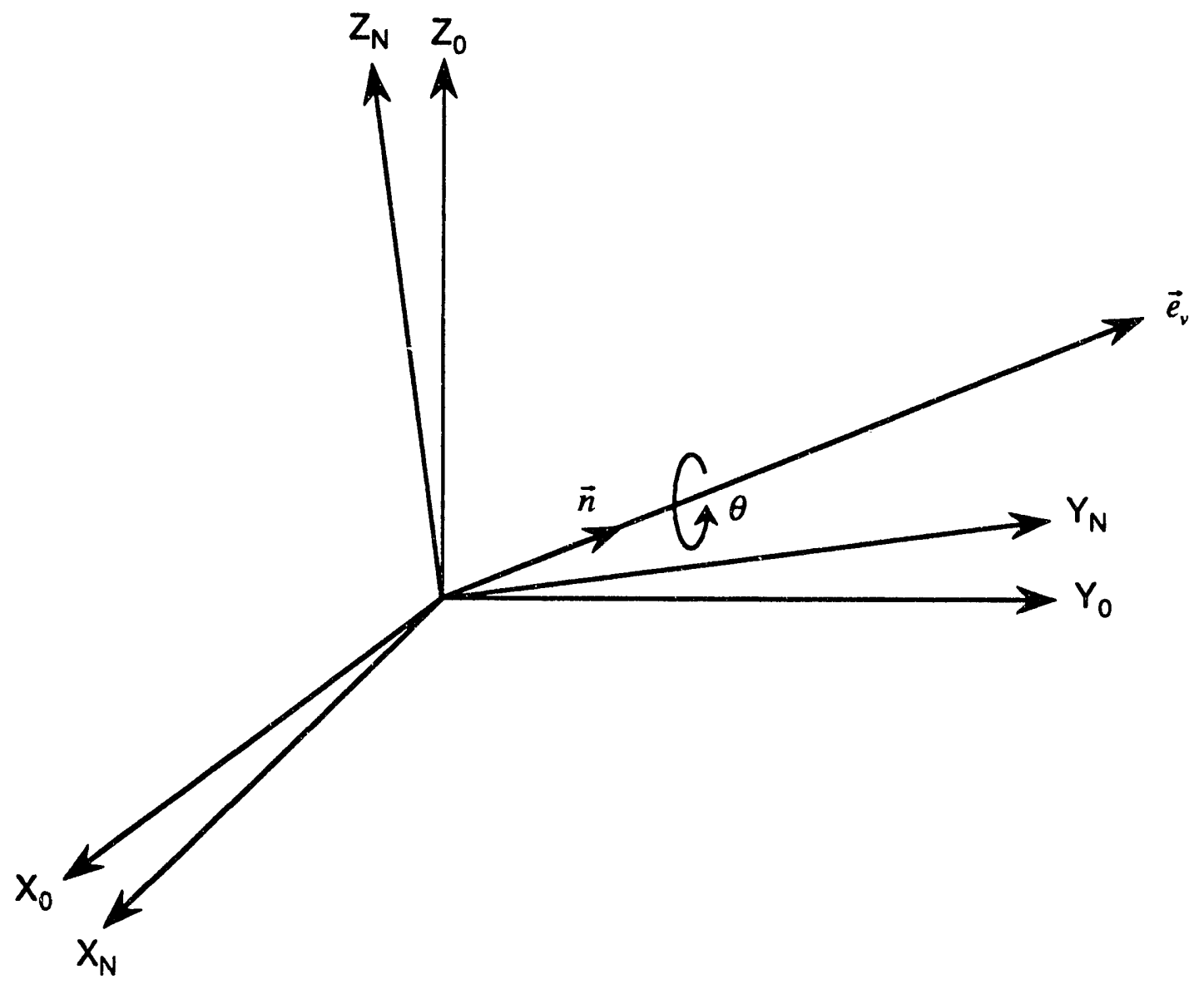

Fig. 2. Rotation of $\left(X_{0}, Y_{0}, Z_{0}\right)$ coordinate frame about unit vector $\vec{n}$ by angle $\theta$ to obtain $\left(X_{N}, Y_{N}, Z_{N}\right)$ coordinate frame. 
normalized quaternions, like the Euler angles, are not a unique representation of orientation. The physical interpretation of eq. (9) is that rotating about the axis $-n$ by angle $-\theta$ is the same as rotating about $n$ by $\theta$. Furthermore, it is straightforward to verify that the following relationships hold:

$$
\begin{aligned}
e\left(n, \pm 360^{\circ}+\theta\right) & =-e(n, \theta), \\
e\left(n, \pm 360^{\circ}-\theta\right) & =-e(n,-\theta)
\end{aligned}
$$

which also demonstrate the nonuniqueness of the normalized quaternions. However, if the rotation is restricted to be about the $+n$ axis and the Euler rotation angle is restricted to the range $\left(-180^{\circ} \leq \theta \leq 180^{\circ}\right)$, then $e_{s} \geq 0$ and $e$ is unique.

A scalar $l$ and Cartesian space vector $r\left(=\left[r_{x}, r_{y}, r_{z}\right]^{T}\right)$ may be represented in the quaternion convention by $\left[l, 0_{1 \times 3}\right]^{T}$ and $\left[0, r^{T}\right]^{T}$, respectively.

\subsection{GENERAL PROPERTIES OF NORMALIZED QUATERNION ALGEBRA}

Let $e^{*}$ denote the quaternion conjugate operation. It is quantified by:

$$
e^{*}=\left[\begin{array}{r}
e_{s} \\
-e_{v}
\end{array}\right] .
$$

Observing Fig. 2 and eq. (6), it is easy to see that $e^{*}$ corresponds to rotating about the Euler axis $n$ by an angle $-\theta$.

Let $e_{1}$ and $e_{2}$ denote two distinct quaternions. The quaternion addition rule is given by:

$$
e_{1}+e_{2}=\left[\begin{array}{c}
e_{1 s}+e_{2 s} \\
e_{1 v}+e_{2 v}
\end{array}\right] .
$$

The quaternion product of $e_{1}$ and $e_{2}$, represented by $e_{1} \circ e_{2}$, can be expressed in a matrix-column vector notation as follows:

$$
e_{1} \circ e_{2}=\Phi\left(e_{1}\right) e_{2}=\Psi\left(e_{2}\right) e_{1}
$$

where $\Phi\left(e_{1}\right)$ and $\Psi\left(e_{2}\right)$ are $(4 \times 4)$ orthogonal matrices defined by [5, 28]:

$$
\begin{aligned}
& \Phi=\left[\begin{array}{cc}
e_{1 s} & -e_{1 v}^{T} \\
e_{1 v} & e_{1 s} I_{3 \times 3}+E\left(e_{1 v}\right)
\end{array}\right], \\
& \Psi=\left[\begin{array}{cc}
e_{2 s} & -e_{2 v}^{T} \\
e_{2 v} & e_{2 s} I_{3 \times 3}-E\left(e_{2 v}\right)
\end{array}\right] .
\end{aligned}
$$


In eqs. (15) and (16), $E\left(e_{1 v}\right)$ and $E\left(e_{2 v}\right)$ are $(3 \times 3)$ matrices arising from expressing the vector cross-product operations $\left(\vec{e}_{1 v} \times \vec{e}_{2 v}\right)$ and $\left(\vec{e}_{2 v} \times \vec{e}_{1 v}\right)$, respectively, in a matrix-column vector notation:

$$
E\left(e_{i v}\right)=\left[\begin{array}{ccc}
0 & -e_{i v z} & e_{i v y} \\
e_{i v z} & 0 & -e_{i v x} \\
-e_{i v y} & e_{i v x} & 0
\end{array}\right]
$$

for $i=1,2$. It can be shown that $\Phi\left(e^{*}\right)=\Phi^{T}(e)$ and $\Psi\left(e^{*}\right)=\Psi^{T}(e)$. Moreover, $e \circ e^{*}=e^{*} \circ e=\left[1,0_{1 \times 3}\right]^{T}$. The quaternion "conjugate" product of $e_{1}$ and $e_{2}$ is represented by $e_{1} \circ e_{2}^{*}$.

\subsection{SUCCESSIVE ROTATIONS AND REPRESENTA- TIONS WITH RESPECT TO VARIOUS COORDI- NATE SYSTEMS}

Suppose the Cartesian coordinate frame $A$ is rotated to obtain coordinate frame $B$. Let quaternions ${ }_{A}^{B} e^{<A>}$ and ${ }_{A}^{B} e^{<0\rangle}$ represent the relative orientation of $B$ with respect to $A$, expressed in the local coordinates $\left(X_{A}, Y_{A}, Z_{A}\right)$ and base coordinates $\left(X_{0}, Y_{0}, Z_{0}\right)$, respectively. Suppose now coordinate frame $B$ is rotated to obtain coordinate frame $C$. The orientation of $C$ with respect to $A$, expressed in terms of the local coordinates, is determined by postmultiplying ${ }_{A}^{B} e^{\langle A\rangle}$ by ${ }_{B}^{C} e^{<B\rangle}[2,3,5,29]$ :

$$
{ }_{A}^{C} e^{\langle A\rangle}={ }_{A}^{B} e^{\langle A\rangle} \circ{ }_{B}^{C} e^{\langle B\rangle} .
$$

On the other hand, the orientation of $C$ with respect to $A$, expressed in terms of the base coordinates, is determined by premultiplying ${ }_{A}^{B} e^{<0\rangle}$ by ${ }_{B}^{C} e^{<0\rangle}[2,3,5,29]$ :

$$
{ }_{A}^{C} e^{<0\rangle}={ }_{B}^{C} e^{\langle 0\rangle} \circ{ }_{A}^{B} e^{\langle 0\rangle} .
$$

Through application of the latter successive rotation formula, it is easy to see that the following relation holds:

$$
{ }_{A}^{C} e^{\langle 0\rangle}=\left[\begin{array}{cc}
1 & 0_{1 \times 3} \\
03 \times 1 & -I_{3 \times 3}
\end{array}\right]{ }_{C}^{A} e^{\langle 0\rangle}={ }_{C}^{A} e^{*\langle 0\rangle} .
$$

Consider the time derivatives of quaternion ${ }_{0}^{A} e^{<0\rangle}$, the orientation of frame $A$ with respect to the base frame, and its conjugate ${ }_{0}^{A} e^{*<0\rangle}$. Applying the theory of coordinate transformations and the relationship between the time derivative of a quaternion and angular velocity of the $A$ th coordinate frame with respect to the zeroth frame, we have $[2,3,5,29]$ :

$$
{ }_{0}^{A} \dot{e}^{\langle 0\rangle}=\frac{1}{2}{ }_{0}^{A} \Omega^{\langle 0\rangle} \circ{ }_{0}^{A} e^{\langle 0\rangle},
$$




$$
{ }_{0}^{A} \dot{e}^{*<0\rangle}=-\frac{1}{2}{ }_{0}^{A} e^{*<0>} \circ{ }_{0}^{A} \Omega^{<0>}
$$

where ${ }_{0}^{A} \Omega^{<0\rangle}$ is a quaternion with scalar part set to zero and vector part equal to the $(3 \times 1)$ Cartesian angular velocity vector $\omega_{0}^{A}$.

For further information on normalized quaternion algebra, the reader is referred to the detailed expositions given in $[30,31]$. The derivation of the orientation errors is discussed in the next section. 


\section{ORIENTATION ERROR ANALYSES}

In this section the orientation error between the desired and actual orientations of the end effector are derived using three methods. The time derivative of the orientation error is then determined, through which a closed loop model of the system is obtain $\_$d. All methods will be expressed in terms of the normalized quaternions. Let the quaternions ${ }_{0}^{N} e^{d<0>}$ and ${ }_{0}^{N} e^{a<0\rangle}(q)$ signify the desired and actual orientations of the end effector coordinate frame $\left(X_{N}, Y_{N}, Z_{N}\right)$ with respect to the base coordinate frame $\left(X_{0}, Y_{0}, Z_{0}\right)$, respectively.

\subsection{APPLICATION OF ICKES ORIENTATION ERROR TO REDUNDANT MANIPULATORS}

This method is based on the previous work of Ickes [7] and Lin [9]. Using the composite quaternion rotation formula given by eq. (19), the orientation error was defined in [7] to be the conjugate product of ${ }_{0}^{N} e^{d<0>}$ and ${ }_{0}^{N} e^{a<0>}$ :

$$
\delta={ }_{0}^{N} e^{d<0>} \circ{ }_{0}^{N} e^{* a<0>}
$$

where ${ }_{0}^{N} e^{* a<0\rangle}$ signifies the quaternion conjugate of ${ }_{0}^{N} e^{a<0\rangle}$ and where $\delta$ is a quaternion expressed in the base coordinates which consists of a scalar component $\delta_{s}$ and a vector component $\delta_{v}$ defined by:

$$
\begin{gathered}
\delta_{s}={ }_{0}^{N} e_{s}^{d<0>}{ }_{0}^{N} e_{s}^{a<0>}+\left({ }_{0}^{N} e_{v}^{d<0>}\right)^{T}{ }_{0}^{N} e_{v}^{a<0>}, \\
\delta_{v}={ }_{0}^{N} e_{v}^{d<0>}{ }_{0}^{N} e_{s}^{a<0>}-{ }_{0}^{N} e_{s}^{d<0>}{ }_{0}^{N} e_{v}^{a<0>}-E\left({ }_{0}^{N} e_{v}^{d<0>}\right)_{0}^{N} e_{v}^{a<0>}
\end{gathered}
$$

in which eqs. (14) and (15) have been applied and where $E\left(\begin{array}{l}N \\ 0\end{array} e_{v}^{d<0>}\right)$ is defined as is $E\left(e_{i v}\right)$ in eq. (17). $\delta_{s}$ and $\delta_{v}$ were termed the scalar and vector components of the quaternion error in [7]. Interestingly, $\delta_{s}=1$ and $\delta_{v}=0_{3 \times 1}$ when the desired and actual orientations of the end effector are identical. $\delta_{v}$ was proposed to be an appropriate representation of the orientation error for tracking control purposes in [7] and has been demonstrated on robotic hardware in $[8,10]$. A rigorous derivation of the orientation error leading to eq. (23) is provided in Appendix B. Noting eq. (7) , it is apparent that the absolute value of any component of $\delta_{v}$ cannot exceed unity and that $\left(\left\|\delta_{v}\right\|_{2}\right)^{2} \leq 1$.

A closed loop error system at the acceleration level for the translational and rotational kinematics of the end effector of a six-axis serial link manipulator employing normalized quaternion feedback was presented in [9]. The error subsystem for the rotational kinematics was obtained by determining the second time derivative of the vector component of the Ickes orientation error quaternion. The procedure in [9] will be adopted here to derive a closed loop model for a redundant manipulator at the velocity level. The analysis in [9], however, assumed that the Jacobian matrix was 
square and directly invertible, which is not the case here. The first time derivative of the vector component of the quaternion error is now considered:

$$
\dot{\delta}_{v}=\left[0_{3 \times 1}, I_{3 \times 3}\right]\left({ }_{0}^{N} \dot{e}^{d} \circ{ }_{0}^{N} e^{* a}+{ }_{0}^{N} e^{d} \circ{ }_{0}^{N} \dot{e}^{* a}\right)
$$

where ${ }_{0}^{N} \dot{\epsilon}^{* a}$ is a function of $\{q, \dot{q}\}$. Since all quaternion algebra and error functions presented throughout this report are expressed in terms of the $\left(X_{0}, Y_{0}, Z_{0}\right)$ coordinate system, the superscript $\langle 0\rangle$ is dropped from eq. (26) and hereinafter for convenience.

Substituting eqs. (21) and (22) into the right hand side of eq. (26) gives:

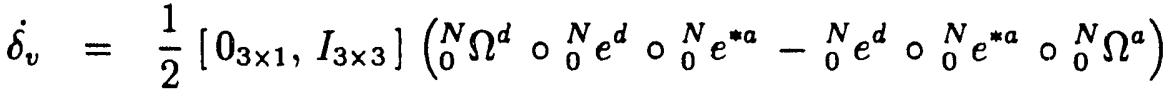

$$
\begin{aligned}
& =\frac{1}{2}\left[0_{3 \times 1}, I_{3 \times 3}\right]\left(\begin{array}{l}
N \\
0
\end{array} \Omega^{d} \circ \delta-\delta \circ{ }_{0}^{N} \Omega^{a}\right)
\end{aligned}
$$

in which eq. (23) has been used and where the normalized quaternions ${ }_{0}^{N} \Omega^{d}$ and ${ }_{0}^{N} \Omega^{a}(q, \dot{q})$ are defined in the same way as ${ }_{0}^{A} \Omega^{<0>}$ below eq. (22).

In Appendix $\mathrm{C}$ it is shown how the quaternion product expressions on the right hand side of eq. (27) can be expressed in a concise matrix-column vector form:

$$
\dot{\delta}_{v}=\frac{1}{2}\left(F^{d} \omega_{0}^{N}-G J_{r} \dot{q}\right)
$$

Our objective is to drive $\dot{\delta}_{v} \rightarrow 0_{3 \times 1}$. To accomplish this, a proportional and integral (PI) feedback control law is introduced:

$$
\dot{\delta}_{v}=-\left(K_{o} \delta_{v}+K_{\text {io }} \int \delta_{v} d t\right)
$$

where $K_{o}$ and $K_{\text {io }}$ are $(3 \times 3)$ positive definite gain matrices.

Eqs. (28) and (29) constitute a closed loop error system for the rotational kinematics of a spatial redundant manipulator. These equations, together with the equations constituting a closed loop error system for the translational kinematics, derived in Appendix D, form a closed loop velocity model for the entire system:

$$
\begin{aligned}
{\left[\begin{array}{c}
{ }^{d} v_{0}^{N} \\
F^{d} \omega_{0}^{N}
\end{array}\right]+} & {\left[\begin{array}{c}
K_{p} p_{e}+K_{i p} \int p_{e} d t \\
2\left(K_{0} \delta_{v}+K_{i o} \int \delta_{v} d t\right)
\end{array}\right] } \\
& =\left[\begin{array}{cc}
I_{3 \times 3} & 0_{3 \times 3} \\
0_{3 \times 3} & G
\end{array}\right] J \dot{q} .
\end{aligned}
$$


To express the model in a form amenable to resolving kinematic redundancy, both sides of eq. (30) are premultiplied by the inverse of the $(6 \times 6)$ coefficient matrix of the Jacobian:

$$
Y_{\text {ickes }}^{\text {in }}=J \dot{q}
$$

where $Y_{i c k e s}^{\text {in }}$ is a $(6 \times 1)$ vector defined by:

$$
Y_{i c k e s}^{\text {in }}=\left[\begin{array}{c}
{ }^{d} v_{0}^{N} \\
G^{-1} F^{d} \omega_{0}^{N}
\end{array}\right]+\left[\begin{array}{c}
K_{p} p_{e}+K_{i p} \int p_{e} d t \\
2 G^{-1}\left(K_{o} \delta_{v}+K_{\text {io }} \int \delta_{v} d t\right)
\end{array}\right] .
$$

In eq. (32), $Y_{\text {ickes }}^{\text {in }}$ is a function of the feedforward variables $\left({ }^{d} p_{0}^{N},{ }^{d} v_{0}^{N},{ }^{d} \omega_{0}^{N},{ }_{0}^{N} e^{d}\right)$ and the feedback variables $\left({ }^{a} p_{0}^{N}(q),{ }_{0}^{N} e^{a}(q)\right)$. When solving eq. (31) for the inverse kinematics, $Y_{i c k e s}^{i n}$ and $J(q)$ are known and $\dot{q}$ is to be determined. This problem will be discussed later in the report.

To arrive at eq. (31), it has been implicitly assumed that matrix $G$ is nonsingular. The conditions under which $G$ is invertible are now discussed. The symbolic determinant of $G$ is given by:

$$
|G|=\delta_{s}\left(\delta_{s}{ }^{2}+\delta_{v x}{ }^{2}+\delta_{v y}{ }^{2}+\delta_{v z}{ }^{2}\right)=\delta_{s}
$$

where eq. (7) has been invoked. Thus $|G|=0$ when $\delta_{s}=0$, which corresponds to the Euler rotation angle taking on the values $\theta= \pm 180^{\circ}$. In this configuration the desired and actual orientations of the end effector are separated by the maximum rotational amount. It is concluded that eq. (31) realistically models the kinematic behavior of the physical manipulator system when the Euler rotation angle is restricted to the range $|\theta|<180^{\circ}$.

The closed loop system proposed in [8] for the rotational kinematics of a manipulator with a proportional control law is equivalent to modeling the system by the equation ${ }^{d} \omega_{0}^{N}-J_{r} \dot{q}=-K_{0} \delta_{v}$ using the notation of this report. We take issue with the approach in [8] because $\delta_{v}$ is not the time integral of the Cartesian angular velocity error expression $\left({ }^{d} \omega_{0}^{N}-J_{r} \dot{q}\right)$. Indeed, ${ }^{d} \omega_{0}^{N}$ and ${ }^{a} \omega_{0}^{N}(q, \dot{q})\left(=J_{r} \dot{q}\right)$ are nonholonomic vectors. The nonintegrable nature of $\left({ }^{d} \omega_{0}^{N}-J_{r} \dot{q}\right)$ is why the time derivative of the vector component of the Ickes orientation error is considered in this report. The control law in eq. (29) is logical and reasonable.

\subsection{APPLICATION OF LWP ORIENTATION ERROR TO REDUNDANT MANIPULATORS}

In 1980 Luh, Walker, and Paul (LWP) conceived an orientation error between the desired and actual end effector attitudes for application to the resolved acceleration 
control of kinematically nonredundant manipulators [11]. Their error function ${ }^{1}$, denoted by $\xi^{l w p}$, is related to the orientation parameters $(n, \theta)$ as follows:

$$
\xi^{l w p}=n \sin (\theta) .
$$

This error function has been studied extensively in the literature $[8,24,32,33]$ and is presently the most widely accepted method for modeling the orientation error in robotics. What has not been considered, however, is the derivation of the analytical time derivative of the LWP orientation error expressed in terms of normalized quaternions, which can be applied to determine a closed loop kinematic velocity model for a redundant manipulator and is the goal of this section.

Eq. (34) can be expressed in an equivalent form:

$$
\xi^{i w p}=2 n \sin (\theta / 2) \cos (\theta / 2) .
$$

Observing the definition of the normalized quaternion $e$ in eq. (6) along with the scalar and vector components of the Ickes orientation error quaternion defined by eqs. (24) and (25) , it follows that the LWP error can be expressed in a normalized quaternion representation:

$$
\xi^{l u p}=2 \delta_{v} \delta_{s} .
$$

Eqs. (7) and (36) reveal that the absolute value of any component of $\xi^{\text {lwp }}$ cannot exceed unity and that $\left(\left\|\xi^{l w p}\right\|_{2}\right)^{2} \leq 1$.

Consider the time derivative of eq. (36):

$$
\dot{\xi}^{l w p}=2\left(\dot{\delta}_{v} \delta_{s}+\delta_{v} \dot{\delta}_{s}\right) \text {. }
$$

A symbolic solution for $\dot{\xi}^{i w p}$ is now sought which is not an explicit function of the variables $\left\{\dot{\delta}_{v}, \dot{\delta}_{s}\right\}$. Examining the structure of the intermediate solution for $\dot{\delta}_{v}$ in eq. (27), it is easy to see that the time derivative of the scalar component of the quaternion error is given by:

$$
\dot{\delta}_{s}=\frac{1}{2}\left[1,0_{1 \times 3}\right]\left({ }_{0}^{N} \Omega^{d} \circ \delta-\delta \circ{ }_{0}^{N} \Omega^{a}\right) .
$$

In Appendix $\mathrm{C}$ it is shown how the two quaternion product expressions on the right hand side of eq. (38) can be expressed in a concise matrix-column vector form:

$$
\dot{\delta}_{s}=-\frac{1}{2} \delta_{v}^{T}\left({ }^{d} \omega_{0}^{N}-J_{r} \dot{q}\right)
$$

Substituting for $\dot{\delta}_{v}$ and $\dot{\delta}_{s}$ in eq. (37) by their definitions in eqs. (28) and (39) obtains:

\footnotetext{
${ }^{1}$ also discussed in $[1$, pg. 165 , eq. $(4,55)]$
} 


$$
\dot{\xi}^{l w_{p}}=\delta_{s}\left(F^{d} \omega_{0}^{N}-G J_{r} \dot{q}\right)-\delta_{v} \delta_{v}{ }^{T}\left({ }^{d} \omega_{0}^{N}-J_{r} \dot{q}\right)
$$

where matrices $F(\delta)$ and $G(\delta)$ are defined by eqs. (C.2) and (C.4), respectively.

A PI feedback control law is introduced to drive $\dot{\xi}^{l w p} \rightarrow 0_{3 \times 1}$ :

$$
\dot{\xi}^{l w p}=-\left(K_{o}^{l} \xi^{l w p}+K_{i o}^{l} \int \xi^{l w p} d t\right)
$$

where $K_{o}^{l}$ and $K_{i o}^{l}$ are $(3 \times 3)$ positive definite gain matrices.

Eqs. (40) and (41) constitute a closed loop error system for the rotational kinematics of a spatial redundant manipulator. These equations, together with the equations constituting a closed loop error system for the translational kinematics, derived in Appendix D, form a closed loop velocity model for the entire system:

$$
\begin{aligned}
{\left[\begin{array}{c}
{ }^{d} v_{0}^{N} \\
\left(\delta_{s} F-\delta_{v} \delta_{v}^{T}\right){ }^{d} \omega_{0}^{N}
\end{array}\right]+\left[\begin{array}{c}
K_{p} p_{e}+K_{i p} \int p_{e} d t \\
K_{o}^{l} \xi^{l w p}+K_{i o}^{l} \int \xi^{l w p} d t
\end{array}\right] } \\
=\left[\begin{array}{cc}
I_{3 \times 3} & 0_{3 \times 3} \\
0_{3 \times 3} & \delta_{s} G-\delta_{v} \delta_{v}^{T}
\end{array}\right] J \dot{q}
\end{aligned}
$$

To express the model in a form amenable to resolving kinematic redundancy, both sides of eq. (42) are premultiplied by the inverse of the $(6 \times 6)$ coefficient matrix of the Jacobian:

$$
Y_{l w p}^{i n}=J \dot{q}
$$

where $Y_{\text {lwp }}^{\text {in }}$ is a $(6 \times 1)$ vector defined by:

$$
\begin{aligned}
Y_{l w p}^{i n}= & {\left[\begin{array}{c}
{ }^{d} v_{0}^{N} \\
\left(\delta_{s} G-\delta_{v} \delta_{v}^{T}\right)^{-1}\left(\delta_{s} F-\delta_{v} \delta_{v}^{T}\right){ }^{d} \omega_{0}^{N}
\end{array}\right] } \\
& +\left[\begin{array}{c}
K_{p} p_{e}+K_{i p} \int p_{e} d t \\
\left(\delta_{s} G-\delta_{v} \delta_{v}^{T}\right)^{-1}\left(K_{o}^{l} \xi^{l w p}+K_{i o}^{l} \int \xi^{l w p} d t\right)
\end{array}\right] .
\end{aligned}
$$

In eq. (44), $Y_{\text {lwp }}^{\text {in }}$ is a function of the feedforward variables $\left({ }^{d} p_{0}^{N},{ }^{d} v_{0}^{N},{ }^{d} \omega_{0}^{N},{ }_{0}^{N} e^{d}\right)$ and the feedback variables $\left({ }^{a} p_{0}^{N}(q),{ }_{0}^{N} e^{a}(q)\right)$. When solving eq. (43) for the inverse kinematics, $Y_{l w p}^{\text {in }}$ and $J(q)$ are known and $\dot{q}$ is to be determined. This problem will be discussed later in the report. 
It has been implicitly assumed that matrix $\left(\delta_{s} G-\delta_{v} \delta_{v}^{T}\right)$ is nonsingular when arriving at eq. (43). The conditions under which this matrix is invertible are now presented. Noting eq. (C.4), this matrix can be expressed as a function of the components of $\delta$ :

$$
\delta_{s} G-\delta_{v} \delta_{v}^{T}=\left[\begin{array}{ccc}
\delta_{s}{ }^{2}-\delta_{v x}{ }^{2} & -\delta_{s} \delta_{v z}-\delta_{v x} \delta_{v y} & \delta_{s} \delta_{v y}-\delta_{v x} \delta_{v z} \\
\delta_{s} \delta_{v z}-\delta_{v x} \delta_{v y} & \delta_{s}{ }^{2}-\delta_{v y}{ }^{2} & -\delta_{s} \delta_{v x}-\delta_{v y} \delta_{v z} \\
-\delta_{s} \delta_{v y}-\delta_{v x} \delta_{v z} & \delta_{s} \delta_{v x}-\delta_{v y} \delta_{v z} & \delta_{s}{ }^{2}-\delta_{v z}{ }^{2}
\end{array}\right]
$$

The determinant of eq. (45) is given by:

$$
\begin{aligned}
\left|\delta_{s} G-\delta_{v} \delta_{v}^{T}\right|= & \delta_{s}{ }^{2}\left\{\delta_{s}{ }^{4}-\delta_{v x}{ }^{4}-\delta_{v y}{ }^{4}-\delta_{v z}{ }^{4}\right. \\
& \left.-2\left(\delta_{v x}{ }^{2} \delta_{v y}{ }^{2}+\delta_{v x}{ }^{2} \delta_{v z}{ }^{2}+\delta_{v y}{ }^{2} \delta_{v z}{ }^{2}\right)\right\}
\end{aligned}
$$

Squaring both sides of the normalized quaternion identity in eq. (7) with $e=\delta$ gives:

$$
\begin{aligned}
1= & \delta_{s}{ }^{4}+\delta_{v x}{ }^{4}+\delta_{v y}{ }^{4}+\delta_{v z}{ }^{4}+2 \delta_{s}{ }^{2}\left(\delta_{v x}{ }^{2}+\delta_{v y}{ }^{2}+\delta_{v z}{ }^{2}\right) \\
& +2\left(\delta_{v x}{ }^{2} \delta_{v y}{ }^{2}+\delta_{v x}{ }^{2} \delta_{v z}{ }^{2}+\delta_{v y}{ }^{2} \delta_{v z}{ }^{2}\right) .
\end{aligned}
$$

Eliminating the term $\left\{-\left(\delta_{v x}{ }^{4}+\delta_{v y}{ }^{4}+\delta_{v z}{ }^{4}\right)\right\}$ on the right hand side of eq. (46) using eq. (47) and simplifying give:

$$
\left|\delta_{s} G-\delta_{v} \delta_{v}^{T}\right|=\delta_{s}{ }^{2}\left\{2 \delta_{s}{ }^{4}-1+2 \delta_{s}{ }^{2}\left(\delta_{v x}{ }^{2}+\delta_{v y}{ }^{2}+\delta_{v z}{ }^{2}\right)\right\} .
$$

Finally, eliminating the term $\left(\delta_{v x}{ }^{2}+\delta_{v y}{ }^{2}+\delta_{v z}{ }^{2}\right)$ in eq. (48) using eq. (7) with $e=\delta$ and simplifying give:

$$
\left|\delta_{s} G-\delta_{v} \delta_{v}^{T}\right|=\delta_{s}{ }^{2}\left(2 \delta_{s}^{2}-1\right) .
$$

Thus $\left|\delta_{s} G-\delta_{v} \delta_{v}^{T}\right|=0$ when $\delta_{s}=0$ or $\delta_{s}= \pm \sqrt{2} / 2$, which correspond to the Euler rotation angle taking on the values $\theta= \pm 180^{\circ}, \pm 90^{\circ}$, respectively. Moreover, the square of the Euclidean norm of the LWP error in fact decreases in value from 1 to 0 when $\theta$ is increased from $90^{\circ}$ to $180^{\circ}$ ( or when $\theta$ decreases from $-90^{\circ}$ to $-180^{\circ}$ ). But intuitively the orientation error should increase as $|\theta|$ increases. It is concluded that eq. (43) realistically models the kinematic behavior of the physical manipulator system when the Euler rotation angle is restricted to the range $|\theta|<90^{\circ}$. Eq. (43) is thought to be the proper closed loop kinematic velocity model to use for resolving the kinematic redundancy when the orientation error is modeled by eq. (36) .

The closed loop system model proposed in [8] for the rotational kinematics based on the LWP error given in eq. (36) with a proportional control law is equivalent to 
modeling the system by the equation ${ }^{d} \omega_{0}^{N}-J_{r} \dot{q}=-2 K_{0}^{l} \delta_{v} \delta_{s}$ using the notation of this report. We take issue with the approach in [8] because $\left(2 \delta_{v} \delta_{s}\right)$ is not the time integral of the Cartesian angular velocity error expression $\left({ }^{d} \omega_{0}^{N}-J_{r} \dot{q}\right)$. The nonholonomic, nonintegrable nature of the angular velocity error is why the time derivative of the LWP error is considered in this report. The control law in eq. (41) is logical and reasonable.

$\xi^{l w p}$ was also expressed in terms of the unit vectors comprising the columns of the desired $\left({ }^{d} R_{0}^{N}\right)$ and actual $\left({ }^{a} R_{0}^{N}(q)\right)$ rotation matrices in [11]. The second time derivative of this alternate form of $\xi^{\text {lwp }}$ was obtained in [33], which led to the determination of a closed loop error subsystem describing the rotational kinematics of a six-axis manipulator at the acceleration level. A kinematic model for the entire nonredundant system was found by combining the rotational error subsystem with the one governing the translational acceleration error. The singularities of the feedback control scheme in [33] are identical to those obtained below eq. (49). The derivation of the closed loop kinematic velocity model in eq. (43) builds on the methods of section 4.1 and exploits the inherent advantages of normalized quaternion feedback.

\subsection{A NEW ERROR METHOD WITH APPLICATION TO REDUNDANT MANIPULATORS}

The minimum number of parameters required to describe the orientation of a rigid body is three [2]. The normalized quaternion parameterization of orientation consists of four components, whose values are restricted by the constraint equation (7). Likewise, the Euler rotation angle and unit vector parameterization is comprised of four components. On the other hand, the Rodrigues parameters are a minimal representation of orientation [2]. It is proposed to model the error between the desired and actual orientations of the end effector of a redundant manipulator by a $(3 \times 1)$ Cartesian space vector $\xi^{\text {rod }}\left(=\left[\xi_{x}^{\text {rod }}, \xi_{y}^{\text {rod }}, \xi_{z}^{\text {rod }}\right]^{T}\right)$ termed the Rodrigues orientation error function. $\xi^{\text {rod }}$ can be defined in terms of the Euler rotation angle and unit vector:

$$
\xi^{\text {rod }}=n \tan (\theta / 2) \text {. }
$$

To apply the unified modeling procedure pressnted in the previous two sections to derive a closed loop kinematic velocity model based on the Rodrigues error function, the right hand side of eq. (50) is expressed in terms of normalized quaternions by invoking eqs. (6), (24), and (25):

$$
\xi^{\text {rod }}=\frac{\delta_{v}}{\delta_{s}}
$$

The denominator on the right hand side of eq. (51) goes to zero when the Euler rotation angle $\theta= \pm 180^{\circ}$, thus the components of the Rodrigues error function can 
become infinite, whereas the absolute value of any component of the Ickes $\left(\delta_{v}\right)$ and LWP $\left(\xi^{\text {lwp }}\right)$ error functions cannot exceed unity. $\xi^{\text {rod }}=0_{3 \times 1}$ when the desired and actual orientations of the end effector are identical.

To derive a closed-loop error system for the rotational kinematics of the redundant manipulator in terms of the Rodrigues orientation error, the time derivative of eq. $(51)$ is now considered:

$$
\dot{\xi}^{\text {rod }}=\frac{\delta_{s} \dot{\delta}_{v}-\delta_{v} \dot{\delta}_{s}}{\delta_{s}^{2}}
$$

Substituting for $\dot{\delta}_{v}$ and $\dot{\delta}_{s}$ in eq. (52) by their definitions in eqs. (28) and (39) give:

$$
\dot{\xi}^{\text {rod }}=\frac{1}{2 \delta_{s}^{2}}\left[\delta_{s}\left(F^{d} \omega_{0}^{N}-G J_{r} \dot{q}\right)+\delta_{v} \delta_{v}^{T}\left({ }^{d} \omega_{0}^{N}-J_{r} \dot{q}\right)\right]
$$

A PI control law is introduced to drive $\dot{\xi}^{\text {rod }} \rightarrow 0_{3 \times 1}$ :

$$
\dot{\xi}^{\text {rod }}=-\left(K_{o}^{r} \xi^{\text {rod }}+K_{i o}^{r} \int \xi^{r o d} d t\right)
$$

where $K_{o}^{r}$ and $K_{i o}^{r}$ are $(3 \times 3)$ positive definite gain matrices.

Eqs. (53) and (54) constitute a closed loop error system for the rotational kinematics of a spatial redundant manipulator. These equations, together with the equations constituting a closed loop error system for the translational kinematics, derived in Appendix D, form a closed loop velocity model for the entire system:

$$
\begin{gathered}
{\left[\begin{array}{c}
{ }^{d} v_{0}^{N} \\
\left(\delta, F+\delta_{v} \delta_{v}{ }^{T}\right){ }^{d} \omega_{0}^{N}
\end{array}\right]+\left[\begin{array}{c}
K_{p} p_{e}+K_{i p} \int p_{e} d t \\
2 \delta_{s}{ }^{2}\left(K_{o}^{r} \xi^{\text {rod }}+K_{i o}^{r} \int \xi^{\text {rod }} d t\right)
\end{array}\right]} \\
=\left[\begin{array}{cc}
I_{3 \times 3} & 0_{3 \times 3} \\
0_{3 \times 3} & \delta_{s} G+\delta_{v} \delta_{v}{ }^{T}
\end{array}\right] J \dot{q}
\end{gathered}
$$

where, here again, matrices $F$ and $G$ are defined by eqs. (C.2) and (C.4), respectively.

To obtain a model amenable to resolving kinematic redundancy, both sides of eq. (55) are premultipled by the inverse of the $(6 \times 6)$ coefficient matrix of the Jacobian:

$$
Y_{\text {rod }}^{\text {in }}=J \dot{q}
$$

where $Y_{\text {rod }}^{\text {in }}$ is a $(6 \times 1)$ vector defined by: 


$$
\begin{aligned}
Y_{\text {rod }}^{\text {in }}= & {\left[\begin{array}{c}
{ }^{d} v_{0}^{N} \\
\left(\delta_{s} G+\delta_{v} \delta_{v}{ }^{T}\right)^{-1}\left(\delta_{s} F+\delta_{v} \delta_{v}{ }^{T}\right){ }^{d} \omega_{0}^{N}
\end{array}\right] } \\
& +\left[\begin{array}{c}
K_{p} p_{e}+K_{i p} \int p_{e} d t \\
2 \delta_{s}{ }^{2}\left(\delta_{s} G+\delta_{v} \delta_{v}{ }^{T}\right)^{-1}\left(K_{0}^{r} \xi^{r o d}+K_{i o}^{r} \int \xi^{r o d} d t\right)
\end{array}\right] .
\end{aligned}
$$

In eq. (57), $Y_{\text {rod }}^{\text {in }}$ is a function of the feedforward variables $\left({ }^{d} p_{0}^{N},{ }^{d} v_{0}^{N},{ }^{d} \omega_{0}^{N},{ }_{0}^{N} e^{d}\right)$ and the feedback variables $\left({ }^{a} p_{0}^{N}(q),{ }_{0}^{N} e^{a}(q)\right)$.

It has been implicitly assumed that matrix $\left(\delta_{s} G+\delta_{v} \delta_{v}{ }^{T}\right)$ is nonsingular when arriving at eq. (56). The conditions under which this matrix is invertible are now presented. Noting eq. (C.4), this matrix can be expressed as a function of the components of $\delta$ :

$$
\delta_{s} G+\delta_{v} \delta_{v}{ }^{T}=\left[\begin{array}{ccc}
\delta_{s}{ }^{2}+\delta_{v x}{ }^{2} & -\delta_{s} \delta_{v z}+\delta_{v x} \delta_{v y} & \delta_{s} \delta_{v y}+\delta_{v x} \delta_{v z} \\
\delta_{s} \delta_{v z}+\delta_{v x} \delta_{v y} & \delta_{s}{ }^{2}+\delta_{v y}{ }^{2} & -\delta_{s} \delta_{v x}+\delta_{v y} \delta_{v z} \\
-\delta_{s} \delta_{v y}+\delta_{v x} \delta_{v z} & \delta_{s} \delta_{v x}+\delta_{v y} \delta_{v z} & \delta_{s}{ }^{2}+\delta_{v z}^{2}
\end{array}\right]
$$

The determinant of eq. (58) is given by:

$$
\begin{aligned}
\left|\delta_{s} G+\delta_{v} \delta_{v}{ }^{T}\right|= & \delta_{s}{ }^{2}\left\{\delta_{s}{ }^{4}+\delta_{v x}{ }^{4}+\delta_{v y}{ }^{4}+\delta_{v z}{ }^{4}\right. \\
& +2\left(\delta_{v x}{ }^{2} \delta_{v y}{ }^{2}+\delta_{v x}{ }^{2} \delta_{v z}{ }^{2}+\delta_{v y}{ }^{2} \delta_{v z}{ }^{2}\right) \\
& \left.+2 \delta_{s}{ }^{2}\left(\delta_{v x}{ }^{2}+\delta_{v y}{ }^{2}+\delta_{v z}{ }^{2}\right)\right\} .
\end{aligned}
$$

Eliminating the expression $\left(\delta_{v x}{ }^{4}+\delta_{v y}{ }^{4}+\delta_{v z}{ }^{4}\right)$ on the right of eq. (59) using eq. (47) and simplifying give:

$$
\left|\delta_{s} G+\delta_{v} \delta_{v}^{T}\right|=\delta_{s}^{2}
$$

Thus $\left|\delta_{s} G+\delta_{v} \delta_{v}^{T}\right|=0$ when $\delta_{s}=0$, which corresponds to the Euler rotation angle taking on the values $\theta= \pm 180^{\circ}$. As anticipated, the singularities of the matrix $\left(\delta_{s} G+\delta_{v} \delta_{v}{ }^{T}\right)$ are identical to the singularities of the Rodrigues orientation error function as discussed below eq. (51). The desired and actual orientations of the end effector are separated by the maximum rotational amount in these singular configurations. It is concluded that eq. (56) realistically models the kinematic behavior of the physical manipulator system when the Euler rotation angle is restricted to the range $|\theta|<180^{\circ}$. Eq. (56) is thought to be the proper closed loop kinematic velocity model to use for resolving the kinematic redundancy when the orientation error is 
modeled by eq. (51). The proposed Rodrigues error function given in eq. (51) is thought to be more sensitive and responsive to orientation errors than the Ickes or LWV error functions. The author is not aware of any previous work on modeling of orientation error using Rodrigues parameters and thus the material in this section is a new result.

The problem of resolving the kinematic redundancy based on the closed loop velocity models derived in this section is discussed next. 


\section{REDUNDANCY RESOLUTION}

In a recent report [34], the input relegation control method was suggested tor resolving the kinematic redundancy of a maninulator based on the model defined by eqs. (3) and (4), which does not take into account the translational and rotational motion tracking control errors. Indeed, this model is open loop in nature and its inputs are the desired, reference trajectories $\left\{{ }^{d} v_{0}^{N},{ }^{d} \omega_{0}^{N}\right\}$ as defined below eq. (4). To overcome this deficiency, the method described in [34] is extended to resolve the redundancy based on any of the three closed loop kinematic velocity models derived here. The basic approach of input relegation control will be discussed without getting irito the details. Additionally, another open loop inverse kinematics algorithm termed the two non-redundant step method is extended to take into accoint the orientation tracking control problem.

\subsection{INPUT RELEGATION CONTROL METHOD}

The closed loop systems described by eqs. (31), (43), and (56) can be expressed in a unified manner by a single equation:

$$
Y_{i}^{i n}=J \dot{q}
$$

where $Y_{i}^{i n}(i=i c k e s, l w p, r o d)$ is a $(6 \times 1)$ vector defined by one of the equations (32), (44), or (57) as selected by the designer.

In input relegation control. a new vector variable $\epsilon=\left[\epsilon_{1}, \epsilon_{2}, \ldots, \epsilon_{N-6}\right]^{T}$ is introducer to resolve the kinematic redundancy. The number of scalar elements contained in $\epsilon$ is equal to the number of redundant DOF contained in the system, namely $(N-6)$. It is defined by:

$$
\epsilon=B \dot{q}
$$

The $((N-6) \times N)$ matrix $B(q)$ in eq. (62) is selected so that the composite $(N \times N)$ matrix $\left(J^{T}, B^{T}\right)^{T}$ is nonsingular. It is convenient to partition the inverse of $\left(J^{T}, B^{T}\right)^{T}$ into two matrices:

$$
\left[\begin{array}{l}
J \\
B
\end{array}\right]^{-1}=[\Pi, \Sigma]
$$

where $\Pi(q)$ is a $(N \times 6)$ matrix and $\Sigma(q)$ a $(N \times(N-6))$ matrix. Eq. (63) implies that $J \Pi=I_{6 \times 6}, J \Sigma=0_{(6 \times(N-6))}, B \Pi=0_{((N-6) \times 6)}, B \Sigma=I_{((N-6) \times(N-6))}$ and $(\Pi J+\Sigma B)=I_{N \times N}$.

Eqs. (61) and (62) can be solved for $\dot{q}$ :

$$
\dot{q}=\Pi Y_{i}^{i n}+\Sigma \epsilon
$$


in which eq. (63) has been invoked. In eq. (64), $\dot{q}$ has been expressed as a function of the variables $\left\{Y_{i}^{i n}, \epsilon, q\right\}$. Substituting the right hand side of eq. (64) into the kinematic velocity model $(61)$ reveals that the expression $(J \Sigma \epsilon)$ identically vanishes regardless of the value of $\epsilon$, since $(J \Sigma)=0_{(6 \times(N-6))}$. Likewise, substituting the right hand side of eq. (64) into eq. (62) reveals that the expression $\left(B \Pi Y_{i}^{\text {in }}\right.$ ) identically vanishes regardless of the value of $Y_{i}^{i n}$, since $(B \Pi)=0_{((N-6) \times 6)}$. Therefore the designer can relegate separate tasks to the quantities $\left\{Y_{i}^{\text {in }}, \epsilon\right\}$.

When applying input relegation control to the orientation error modeling and control framework presented here, it is assumed that the desired trajectories of the variables $\left\{{ }^{d} p_{0}^{N},{ }^{d} v_{0}^{N},{ }^{d} \omega_{0}^{N},{ }_{0}^{N} e^{d}\right\}$ have been specified by the designer and the actual values of $\left\{{ }^{a} p_{0}^{N}(q),{ }_{0}^{N} e^{a}(q)\right\}$ are available via feedback loops. There is now sufficient information available to calculate $Y_{i}^{i n}(i=i c k e s, l w p$, rod $)$ using one of the equations (32), (44), or (57) as selected by the designer. It is also assumed that $J(q)$ is known. To calculate the generalized velocities using eq. (64), the values of $\{\Pi, \Sigma, \epsilon\}$ need to be determined. In [34], it is assumed that the designer first selects matrix $B$ such that $\left(J^{T}, B^{T}\right)^{T}$ is nonsingular, which immediately leads to the determination of $(\Pi, \Sigma)$ by eq. (63) . Several techniques for selecting $B$ are discussed in [34], one of which is described later in this section. However, eq. (64) still cannot be solved for $\dot{q}$ since $\epsilon$ is an unknown quantity. To solve for $\{\epsilon, \dot{q}\}$, an optimization scheme was suggested in [34] to pick $\epsilon$ to secure a minimum Euclidean norm solution for the generalized velocities. The solutions for these quantities based on eq. (64) are given by:

$$
\begin{gathered}
\epsilon=-\left(\Sigma^{T} \Sigma\right)^{-1} \Sigma^{T} \Pi Y_{i}^{i n} \\
\dot{q}=\left(I_{N \times N}-\Sigma\left(\Sigma^{T} \Sigma\right)^{-1} \Sigma^{T}\right) \Pi Y_{i}^{i n} .
\end{gathered}
$$

An analytical method was presented in [34] to choose $B$ to maximize the determinant of matrix $\left(J^{T}, B^{T}\right)^{T}$ with the restriction that $B$ is orthogonal to the rows of $J$, i.e., $J B^{T}=0_{6 \times 1}$, for manipulators with one degree of redundancy. Postmuitiplying the matrix identity $\Pi J+\Sigma B=I_{7 \times 7}$ by $J^{T}$ immediately leads to a symbolic solution for $\Pi$ :

$$
\Pi=J^{T}\left(J J^{T}\right)^{-1}
$$

Furthermore, $J B^{T}=0_{6 \times 1}$ implies that $\Pi^{T} \Sigma=0_{6 \times 1}$ [34]. Eqs. (65) and (66) immediately simplify to:

$$
\begin{gathered}
\epsilon=0, \\
\dot{q}=J^{T}\left(J J^{T}\right)^{-1} Y_{i}^{i n} .
\end{gathered}
$$

A numerically efficient method for calculating eq. (69) in two steps is discussed next. 


\subsection{TWO NON-REDUNDANT STEP METHOD}

This method is based on the previous work [18] which calculated the Moore-Penrose pseudoinverse of the Jacobian matrix in two "non-redundant" steps to yield a minimum norm solution for $\dot{q}$ based on the open loop model given by eq. (3). The method can be extended to calculate a solution for $\dot{q}$ based on the closed loop system with normalized quaternion feedback given by eq. (61). In the first step, the following equation is solved for $W$, a $(6 \times 1)$ vector:

$$
J J^{T} W=Y_{i}^{\text {in }} .
$$

The generalized velocities are then obtained in the second step:

$$
\dot{q}=J^{T} W .
$$

The explicit inversion of the $(6 \times 6)$ matrix $\left(J J^{T}\right)$ in the symbolic solution for

$W=\left(J J^{T}\right)^{-1} Y_{i}^{\text {in }}$ may be avoided by applying the numerical $L U$ decomposition method for solving systems of linear algebraic equations [35].

Interestingly, an inverse kinematics scheme based on eq. (61) can be implemented in an open loop or closed loop manner to the physical plant, i.e., the manipulator. In an open loop implementation, the translational position and normalized quaternion feedback loops are taken from the output of the redundancy resolution scheme, as shown in Fig. 3. This approach assumes that the lower level joint or encoder space feedback control laws can track the commanded articulated joint or encoder trajectories perfectly. In Fig. 3, the superscript a means the variable is calculated using the articulated, commanded values of the generalized coordinates available at the output of the redundancy resolution scheme. In a closed loop implementation of redundancy resolution, the feedback loops are taken from the output of the physical plant, as shown in Fig. 4. In this case the algorithm resolves the redundancy based on feedback measurements of sensors, e.g., the encoder sensors of the motors of the CESARm research manipulator $[21,36,37,38]$. A comparison of the performance of the input relegation control using eq. (61) when implemented in an open or closed loop manner to the plant is currently being investigated. 


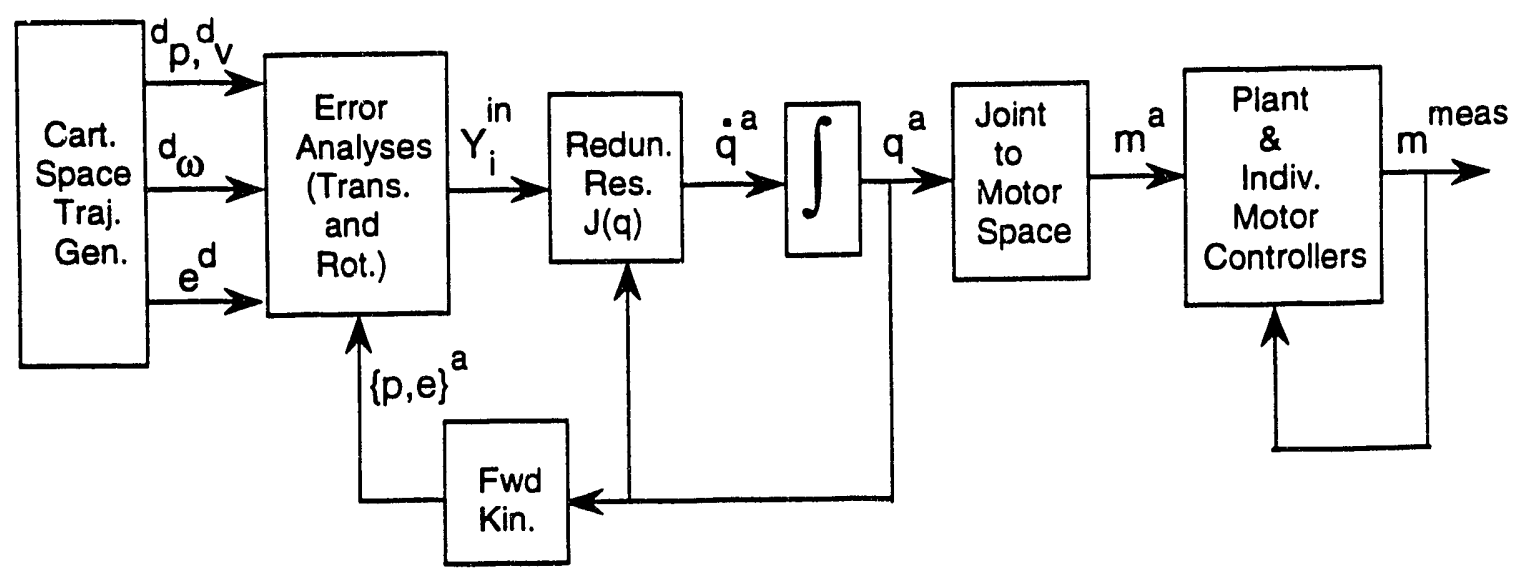

Fig. 3. Open loop redundancy resolution to the plant.

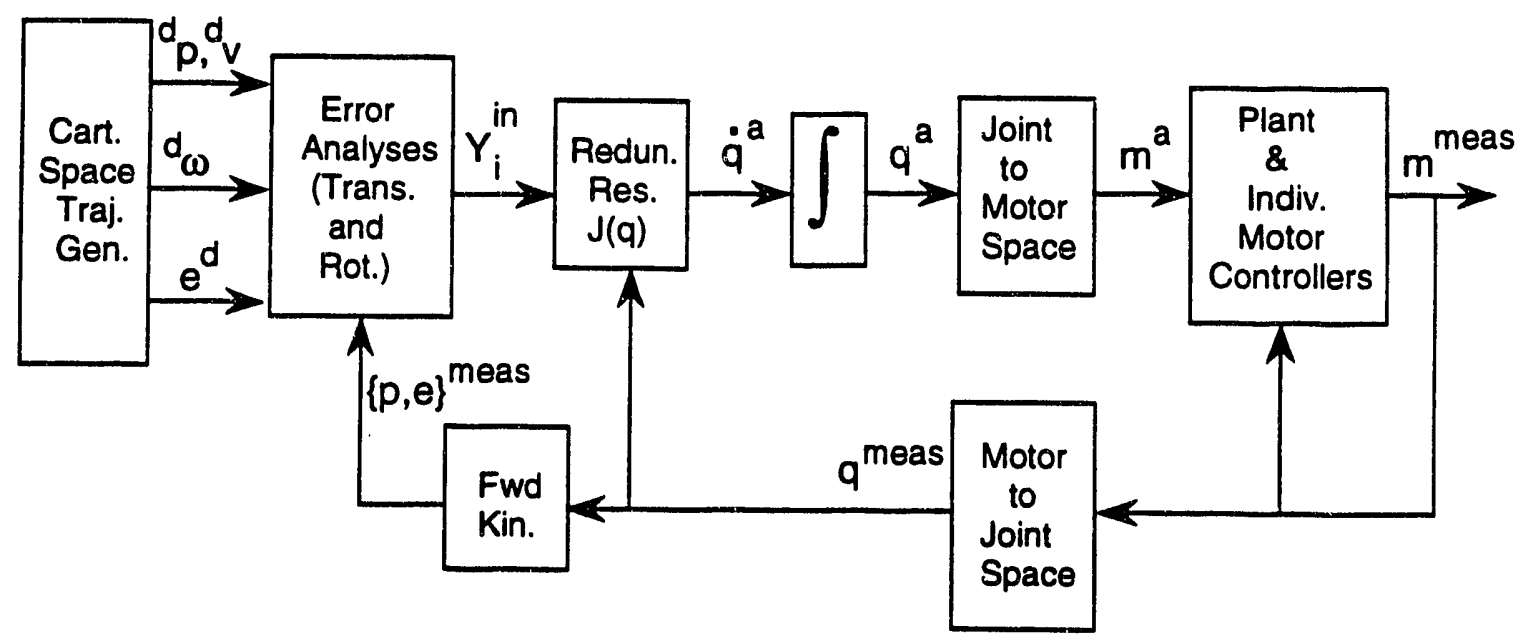

Fig. 4. Closed loop redundancy resolution to the plant. 


\section{CONCLUSION AND FUTURE WORK}

The error between the desired and actual orientations of the end effector of a spatial kinematically redundant autonomous manipulator was modeled in a normalized quaternion algebra framework by three different methods: (i) the vector component of the Ickes orientation error quaternion; (ii) the Luh, Walker, Paul (LWP) method; and (iii) a new method based on the Rodrigues parameters. It was shown that the LWP and Rodrigues errors can be modeled as explicit functions of the scalar and vector components of the Ickes orientation error quaternion. The time derivative of each of the orientation errors was derived symbolically and combined with the translational velocity error expression to yield a distinct closed loop kinematic velocity model employing normalized quaternion and translational position feedback. The singularities of the coefficient matrix which premultiplies the $(6 \times N)$ Jacobian matrix $J(q)$ in each model were determined analytically, because the coefficient matrix had to be inverted to express each model in a form suitable for solving the inverse kinematics problem. It was found that singularities occurred when the Euler rotation angle $(\theta)$ took on the values $\pm 180^{\circ}$ when deriving closed loop models by all three methods, with the LWP error based method possessing additional singularities at $\theta= \pm 90^{\circ}$. It was concluded that the Euler Rotation Angle must be restricted to the range $|\theta|<180^{\circ}$ for the Ickes and Rodrigues error methods, and to $|\theta|<90^{\circ}$ for the LWP error method.

The report argued that the normalized quaternions are superior to Euler angles for modeling the orientation error in a kinematically redundant manipulator. This is because the time derivatives of the desired and actual normalized quaternions $\left\{{ }_{0}^{N} \dot{e}^{d},{ }_{0}^{N} \dot{e}^{a}\right\}$ and their conjugates $\left\{{ }_{0}^{N} \dot{e}^{* d},{ }_{0}^{N} \dot{e}^{* a}\right\}$ can be expressed by singularity free functions of the quaternions $\left\{{ }_{0}^{N} e^{d},{ }_{0}^{N} e^{a}(q)\right\}$ and their conjugates $\left\{{ }_{0}^{N} e^{* d},{ }_{0}^{N} e^{* a}(q)\right\}$, respectively, as well as the desired and actual angular velocities $\left\{{ }^{d} \omega_{0}^{N},{ }^{a} \omega_{0}^{N}(q, \dot{q})\right\}$ using eqs. (21) and (22) .

On the other hand, inherent singularities arise when expressing the time derivatives of the Euler angles as functions of the Euler angles and the Cartesian angular velocity. It has been shown that the identical singularities arise when expressing Jacobian $J^{*}$ as a function of $J$ and the Euler angles, or when extracting the Euler angles from an orthogonal rotation matrix for feedback control purposes. In particular, these singularities can happen when the designer uses eq. (2) to resolve the kinematic redundancy. Likewise, eq. (3) is ruled out as a basis for inverse kinematics of a spatial redundant manipulator where the orientation of the end effector is to be controlled to track a reference trajectory because the Cartesian angular velocity error expression $\left({ }^{d} \omega_{0}^{N}-J_{r} \dot{q}\right)$ is a nonholonomic, nonintegrable quantity. The modeling presented here is an explicit function of Jacobian $J$ which can be calculated in a singularity free manner. We conclude that the three models presented here employing normalized quaternion feedback are more appropriate for inverse kinematics applica- 
tions where the spatial orientation tracking control problem is to be accounted for than the models in eqs. (2) and (3) .

The report demonstrated how the input relegation control and two nonredundant step inverse kinematic algorithms could be extended to properly take into account the spatial orientation tracking control problem based on the modeling given in this report. It should be mentioned that the theory presented here has been experimentally verified on the CESARm research manipulator by the author and the results will be published in future papers.

The research presented in this report has uncovered and identified a wealth of open research issues that warrant future attention. To conduct an experimental comparison of the three orientation error methods discussed in this report would be a very worthwhile and beneficial way to ascertain their merits relative to one another. This research could include a comparison of performances when the inverse kinematics is implemented in open and closed loop manners to the plant, as illustrated in Figs. 3 and 4.

It would be of interest to investigate the stability of the redundant manipulator system based on the three closed loop kinematic velocity models derived here. The results of the stability analyses would complement those of the singularity analyses in determining which of the three orientation tracking control schemes is superior.

Another suggested future research topic is to extend the approach given here to derive a closed loop kinematic acceleration model employing normalized quaternion feedback for each of the three orientation error methods. Such a model could be combined with the equations of motion of the manipulator to optimize a dynamic model based criteria when resolving the redundancy. 


\section{ACKNOWLEDGMENT}

This research was supported in part by the Engineering Research Program, Office of Basic Energy Sciences, U.S. Department of Energy, and in part by the Office of Technology Support Programs, Office of Nuclear Energy, U.S. Department of Energy, under Contract No. DE-AC05-840R21400 with Martin Marietta Energy Systems, Inc. The author wishes to thank Dr. J.F. Jansen and Dr. R.L. Kress for reviewing this report and providing many insightful suggestions for its improvement. 


\section{APPENDIX A SINGULARITIES IN COMPUTING EULER ANGLES, THEIR TIME DERIVATIVES, AND JACOBIAN $J^{*}(q)$}

In this Appendix we review the singularities associated with a well known algorithm devised to extract the Euler angles $\{\alpha, \beta, \gamma\}$ from an orthogonal rotation matrix ${ }^{a} R_{0}^{N}(q)$ [6] for the XYZ, ZYZ, and ZXZ conventions. We also review the singularities associated with calculating the time derivatives of the Euler angles as linear functions of the Cartesian angular velocity of the end effector ${ }^{a} \omega_{0}^{N}(q, \dot{q})$ with respect to the $\left(X_{0}, Y_{0}, Z_{0}\right)$ coordinate frame [24, 26, 10]. Finally, the singularities arising from calculating Jacobian $J^{*}(q)$ as a function of the Jacobian $J(q)$ and the Euler angles are demonstrated. To express the orientation of the end effector coordinate frame $\left(X_{N}, Y_{N}, Z_{N}\right)$ with respect to $\left(X_{0}, Y_{0}, Z_{0}\right)$, it is convenient to assume that the origins of these frames coincide and to let $\left(X^{\prime}, Y^{\prime}, Z^{\prime}\right)$ and $\left(X^{\prime \prime}, Y^{\prime \prime}, Z^{\prime \prime}\right)$ represent intermediate coordinate systems. It is useful to define ${ }^{a} R_{0}^{N}$ in terms of its elements:

$$
{ }^{a} R_{0}^{N}=\left[\begin{array}{lll}
r_{11} & r_{12} & r_{13} \\
r_{21} & r_{22} & r_{23} \\
r_{31} & r_{32} & r_{33}
\end{array}\right]
$$

\section{XYZ CONVENTION EULER ANGLES}

Rotate about $Z_{0}$ by angle $\alpha$ to obtain $\left(X^{\prime}, Y^{\prime}, Z^{\prime}\right)$. Rotate about $Y^{\prime}$ by angle $\beta$ to obtain $\left(X^{\prime \prime}, Y^{\prime \prime}, Z^{\prime \prime}\right)$. Finally, rotate about $X^{\prime \prime}$ by angle $\gamma$ to obtain $\left(X_{N}, Y_{N}, Z_{N}\right)$. ${ }^{a} R_{0}^{N}(\alpha, \beta, \gamma)$ is given by $[1,6]$ :

$$
{ }^{a} R_{0}^{N}=\left[\begin{array}{ccc}
c(\beta) c(\alpha), & s(\gamma) s(\beta) c(\alpha)-c(\gamma) s(\alpha), & c(\gamma) s(\beta) c(\alpha)+s(\gamma) s(\alpha) \\
c(\beta) s(\alpha), & s(\gamma) s(\beta) s(\alpha)+c(\gamma) c(\alpha), & c(\gamma) s(\beta) s(\alpha)-s(\gamma) c(\alpha) \\
-s(\beta) & c(\beta) s(\gamma) & c(\beta) c(\gamma)
\end{array}\right]
$$

where $c(\cdot)=\cos (\cdot)$ and $s(\cdot)=\sin (\cdot)$.

The Euler angles can be extracted from eq. (A.2) [6]:

$$
\beta=\operatorname{Atan} 2\left(-r_{31}, \sqrt{r_{11}^{2}+r_{21}^{2}}\right)
$$

or

$$
\begin{aligned}
& \beta=\operatorname{Atan} 2\left(-r_{31}, \sqrt{r_{32}^{2}+r_{33}^{2}}\right), \\
& \alpha=\operatorname{Atan} 2\left(r_{21} / c(\beta), r_{11} / c(\beta)\right),
\end{aligned}
$$




$$
\gamma=\operatorname{Atan} 2\left(r_{32} / c(\beta), r_{33} / c(\beta)\right) .
$$

The above algorithm degenerates when:

$$
c(\beta)=0, \quad \beta= \pm 90^{\circ} .
$$

It is well known that ${ }^{a} \omega_{0}^{N}$ can be expressed as a function of the Euler angles and their time derivatives [1]:

$$
{ }^{a} \omega_{0}^{N}=\left[\begin{array}{ccc}
0 & -s(\alpha) & c(\alpha) c(\beta) \\
0 & c(\alpha) & s(\alpha) c(\beta) \\
1 & 0 & -s(\beta)
\end{array}\right]\left[\begin{array}{c}
\dot{\alpha} \\
\dot{\beta} \\
\dot{\gamma}
\end{array}\right]
$$

A solution for $[\dot{\alpha}, \dot{\beta}, \dot{\gamma}]$ can be obtained from eq. (A.8) $[24,26,10]$ :

$$
\left[\begin{array}{c}
\dot{\alpha} \\
\dot{\beta} \\
\dot{\gamma}
\end{array}\right]=-\frac{1}{c(\beta)}\left[\begin{array}{ccc}
-c(\alpha) s(\beta) & -s(\alpha) s(\beta) & -c(\beta) \\
s(\alpha) c(\beta) & -c(\alpha) c(\beta) & 0 \\
-c(\alpha) & -s(\alpha) & 0
\end{array}\right]{ }^{a} \omega_{0}^{N}
$$

Using eqs. (2) and (3), it is straightforward to verify that the Jacobian $J^{*}$ can be expressed as a function of the Jacobian $J$ and $[\alpha, \beta, \gamma]$ :

$$
J^{*}=\left[\begin{array}{ccc}
I_{3 \times 3} & 0_{3 \times 3} \\
& -\frac{1}{c(\beta)}\left[\begin{array}{ccc}
-c(\alpha) s(\beta) & -s(\alpha) s(\beta) & -c(\beta) \\
s(\alpha) c(\beta) & -c(\alpha) c(\beta) & 0 \\
-c(\alpha) & -s(\alpha) & 0
\end{array}\right]
\end{array}\right] J
$$

It is easy to see that a singularity occurs in the above two equations under the conditions given in eq. (A.7) .

\section{ZYZ CONVENTION EULER ANGLES}

Rotate about $Z_{0}$ by $\alpha$ to obtain $\left(X^{\prime}, Y^{\prime}, Z^{\prime}\right)$. Rotate about $Y^{\prime}$ by $\beta$ to obtain $\left(X^{\prime \prime}, Y^{\prime \prime}, Z^{\prime \prime}\right)$. Finally, rotate about $Z^{\prime \prime}$ by $\gamma$ to obtain $\left(X_{N}, Y_{N}, Z_{N}\right) \cdot{ }^{a} R_{0}^{N}(\alpha, \beta, \gamma)$ is given by $[1,6,39]$ :

$$
{ }^{a} R_{0}^{N}=\left[\begin{array}{ccc}
-s(\gamma) s(\alpha)+c(\beta) c(\alpha) c(\gamma), & -c(\gamma) s(\alpha)-c(\beta) c(\alpha) s(\gamma), & s(\beta) c(\alpha) \\
s(\gamma) c(\alpha)+c(\beta) s(\alpha) c(\gamma), & c(\gamma) c(\alpha)-c(\beta) s(\alpha) s(\gamma), & s(\beta) s(\alpha) \\
-c(\gamma) s(\beta) & s(\gamma) s(\beta) & c(\beta)
\end{array}\right]
$$

The Euler angles can be extracted from eq. (A.11) [6]:

$$
\beta=\operatorname{Atan} 2\left(\sqrt{r_{31}^{2}+r_{32}^{2}}, r_{33}\right)
$$


or

$$
\begin{gathered}
\beta=\operatorname{Atan} 2\left(\sqrt{r_{13}^{2}+r_{23}^{2}}, r_{33}\right), \\
\alpha=\operatorname{Atan} 2\left(r_{23} / s(\beta), r_{13} / s(\beta)\right), \\
\gamma=\operatorname{Atan} 2\left(r_{32} / s(\beta),-r_{31} / s(\beta)\right) .
\end{gathered}
$$

The above algorithm degenerates when:

$$
s(\beta)=0, \quad \beta=0, \pm 180^{\circ} .
$$

It is well known that ${ }^{a} \omega_{0}^{N}$ can be expressed as a function of the Euler angles and their time derivatives [1]:

$$
{ }^{a} \omega_{0}^{N}=\left[\begin{array}{ccc}
0 & -s(\alpha) & c(\alpha) s(\beta) \\
0 & c(\alpha) & s(\alpha) s(\beta) \\
1 & 0 & c(\beta)
\end{array}\right]\left[\begin{array}{c}
\dot{\alpha} \\
\dot{\beta} \\
\dot{\gamma}
\end{array}\right]
$$

A solution for $[\dot{\alpha}, \dot{\beta}, \dot{\gamma}]$ can be obtained from eq. (A.17) $[24,26,10]$ :

$$
\left[\begin{array}{c}
\dot{\alpha} \\
\dot{\beta} \\
\dot{\gamma}
\end{array}\right]=-\frac{1}{s(\beta)}\left[\begin{array}{ccc}
c(\alpha) c(\beta) & s(\alpha) c(\beta) & -s(\beta) \\
s(\alpha) s(\beta) & -c(\alpha) s(\beta) & 0 \\
-c(\alpha) & -s(\alpha) & 0
\end{array}\right] a \omega_{0}^{N}
$$

$J^{*}$ can be expressed as a function of the $J$ and $[\alpha, \beta, \gamma]$ :

$$
J^{*}=\left[\begin{array}{ccc}
I_{3 \times 3} & 0_{3 \times 3} \\
& -\frac{1}{s(\beta)}\left[\begin{array}{ccc}
c(\alpha) c(\beta) & s(\alpha) c(\beta) & -s(\beta) \\
s(\alpha) s(\beta) & -c(\alpha) s(\beta) & 0 \\
-c(\alpha) & -s(\alpha) & 0
\end{array}\right]
\end{array}\right] J
$$

It is easy to see that a singularity occurs in the above two equations under the conditions given in eq. (A.16) .

\section{ZXZ CONVENTION EULER ANGLES}

Rotate about $Z_{0}$ by $\alpha$ to obtain $\left(X^{\prime}, Y^{\prime}, Z^{\prime}\right)$. Rotate about $X^{\prime}$ by $\beta$ to obtain $\left(X^{\prime \prime}, Y^{\prime \prime}, Z^{\prime \prime}\right)$. Finally, rotate about $Z^{\prime \prime}$ by $\gamma$ to obtain $\left(X_{N}, Y_{N}, Z_{N}\right) .{ }^{a} R_{0}^{N}(\alpha, \beta, \gamma)$ is given by [1]:

$$
{ }^{a} R_{0}^{N}=\left[\begin{array}{ccc}
c(\gamma) c(\alpha)-c(\beta) s(\alpha) s(\gamma), & -s(\gamma) c(\alpha)-c(\beta) s(\alpha) c(\gamma), & s(\beta) s(\alpha) \\
c(\gamma) s(\alpha)+c(\beta) c(\alpha) s(\gamma), & -s(\gamma) s(\alpha)+c(\beta) c(\alpha) c(\gamma), & -s(\beta) c(\alpha) \\
s(\beta) s(\gamma) & s(\beta) c(\gamma) & c(\beta)
\end{array}\right]
$$


The Euler angles can be extracted from eq. (A.20) [6]:

or

$$
\beta=\operatorname{Atan} 2\left(\sqrt{r_{31}^{2}+r_{32}^{2}}, r_{33}\right)
$$

$$
\begin{gathered}
\beta=\operatorname{Atan} 2\left(\sqrt{r_{13}^{2}+r_{23}^{2}}, r_{33}\right), \\
\alpha=\operatorname{Atan} 2\left(r_{13} / s(\beta),-r_{23} / s(\beta)\right), \\
\gamma=\operatorname{Atan} 2\left(r_{31} / s(\beta), r_{32} / s(\beta)\right) .
\end{gathered}
$$

The above algorithm degenerates under conditions described in eq. (A.16)

It is well known that ${ }^{a} \omega_{0}^{N}$ can be expressed as a function of the Euler angles and their time derivatives $[1,39]$ :

$$
{ }^{a} \omega_{0}^{N}=\left[\begin{array}{ccc}
0 & c(\alpha) & s(\alpha) s(\beta) \\
0 & s(\alpha) & -c(\alpha) s(\beta) \\
1 & 0 & c(\beta)
\end{array}\right]\left[\begin{array}{c}
\dot{\alpha} \\
\dot{\beta} \\
\dot{\gamma}
\end{array}\right] .
$$

A solution for $[\dot{\alpha}, \dot{\beta}, \dot{\gamma}]$ can be obtained from eq. (A.25) $[24,26,10]$ :

$$
\left[\begin{array}{c}
\dot{\alpha} \\
\dot{\beta} \\
\dot{\gamma}
\end{array}\right]=-\frac{1}{s(\beta)}\left[\begin{array}{ccc}
s(\alpha) c(\beta) & -c(\alpha) c(\beta) & -s(\beta) \\
-c(\alpha) s(\beta) & -s(\alpha) s(\beta) & 0 \\
-s(\alpha) & c(\alpha) & 0
\end{array}\right] a \omega_{0}^{N} .
$$

$J^{*}$ can be expressed as a function of the $J$ and $[\alpha, \beta, \gamma]$ :

$$
J^{*}=\left[\begin{array}{ccc}
I_{3 \times 3} & 0_{3 \times 3} & \\
& \\
0_{3 \times 3} & -\frac{1}{s(\beta)}\left[\begin{array}{ccc}
s(\alpha) c(\beta) & -c(\alpha) c(\beta) & -s(\beta) \\
-c(\alpha) s(\beta) & -s(\alpha) s(\beta) & 0 \\
-s(\alpha) & c(\alpha) & 0
\end{array}\right]
\end{array}\right] J
$$

It is easy to see that a singularity occurs in the above two equations under the conditions given in eq. (A.16) .

It has been shown that the singularities associated with extracting Euler angles from ${ }^{a} R_{0}^{N}$ and calculating $[\dot{\alpha}, \dot{\beta}, \dot{\gamma}]$ as a linear function of ${ }^{a} \omega_{0}^{N}$ are identical for each of the three conventions. 


\section{APPENDIX B DERIVATION OF ICKES ORIENTATION ERROR}

In this Appendix the analytical expression for the error between the desired and actual end effector orientations proposed by Ickes is derived based on the successive rotation formula for normalized quaternions. Let ${ }_{a}^{d} e^{<0\rangle}$ be the quaternion representation of the error between the desired ${ }_{0}^{d} e^{\langle 0\rangle}$ and actual ${ }_{0}^{a} e^{\langle 0\rangle}(q)$ orientations of the end effector, expressed in terms of the $\left(X_{0}, Y_{0}, Z_{0}\right)$ coordinate system. Based on eq. (19), it is straightforward to verify that the following relation holds:

$$
{ }_{0}^{d} e^{\langle 0\rangle}={ }_{a}^{d} e^{\langle 0\rangle} \circ{ }_{0}^{a} e^{\langle 0\rangle} .
$$

The problem is to solve eq. (B.1) for ${ }_{a}^{d} e^{\langle 0\rangle}$, where it is assumed that ${ }_{0}^{d} e^{\langle 0\rangle}$ and ${ }_{0}^{a} e^{<0>}$ are known. Eq. (B.1) can be expressed in a matrix-column vector form:

$$
{ }_{0}^{d} e^{\langle 0\rangle}=\Psi\left({ }_{0}^{a} e^{\langle 0\rangle}\right){ }_{a}^{d} e^{<0\rangle}
$$

in which eq. (14) has been invoked. Since $\Psi\left({ }_{0}^{a} e^{<0>}\right)$ is an orthogonal macrix, we have:

$$
\begin{aligned}
{ }_{a}^{d} e^{\langle 0\rangle} & =\left(\Psi\left({ }_{0}^{a} e^{\langle 0\rangle}\right)\right)_{0}^{T}{ }_{0}^{d} e^{\langle 0\rangle} \\
& =\Psi\left({ }_{0}^{a} e^{m\langle 0\rangle}\right){ }_{0}^{d} e^{\langle 0\rangle} .
\end{aligned}
$$

Eq. (B.3) can be expressed in terms of the quaternion product notation:

$$
{ }_{a}^{d} e^{\langle 0\rangle}={ }_{0}^{d} e^{\langle 0\rangle} \circ{ }_{0}^{a} e^{*\langle 0\rangle}
$$

in which eq. (14) has been used. Applying eq. (B.4) to a redundant manipulator yields eq. (23).

It is of interest to derive the orientation error ${ }_{d}^{a} e^{\langle 0\rangle}$, which is the actual end effector orientation relative to desired orientation, again expressed in the base coordinates. Applying eq. (19), the following relation holds:

$$
\begin{aligned}
{ }_{0}^{a} e^{\langle 0\rangle} & ={ }_{d}^{a} e^{\langle 0\rangle} \circ{ }_{0}^{d} e^{\langle 0\rangle} \\
& =\Psi\left({ }_{0}^{d} e^{\langle 0\rangle}\right){ }_{d}^{a} e^{\langle 0\rangle} .
\end{aligned}
$$

Solving eq. (B.5) yields:

$$
\begin{aligned}
{ }_{d}^{a} e^{\langle 0\rangle} & =\Psi\left({ }_{0}^{d} e^{*\langle 0\rangle}\right){ }_{0}^{a} e^{\langle 0\rangle} \\
& ={ }_{0}^{a} e^{\langle 0\rangle} \circ{ }_{0}^{d} e^{*\langle 0\rangle} .
\end{aligned}
$$


Carrying through the multiplications in eqs. (B.4) and (B.6), it can be shown that:

$$
{ }_{d}^{a} e^{\langle 0\rangle}=\left[\begin{array}{cc}
1 & 0_{1 \times 3} \\
03 \times 1 & -I_{3 \times 3}
\end{array}\right]{ }_{a}^{d} e^{\langle 0\rangle}={ }_{a}^{d} e^{*<0\rangle}
$$

as expected, where, here again, $I_{r \times r}$ denotes an $(r \times r)$ identity matrix and $0_{r \times s}$ an $(r \times s)$ matrix of zeros. 


\section{APPENDIX C \\ QUATERNION PRODUCT SIMPLIFICATIONS}

In this Appendix two quaternion product expressions appearing in the derivations of the time derivatives of the orientation error are reduced to a simpler form.

\section{Part C1}

The first quaternion product expression on the right hand side of eq. (27) can be replaced by its equivalent matrix-column vector representation:

$$
\begin{aligned}
{ }_{0}^{N} \Omega^{d} \circ \delta & =\left[\begin{array}{cc}
\delta_{s} & -\delta_{v}^{T} \\
\delta_{v} & F
\end{array}\right]\left[\begin{array}{c}
0 \\
{ }_{d} \omega_{0}^{N}
\end{array}\right] \\
& =\left[\begin{array}{c}
-\delta_{v}{ }^{T} \\
F
\end{array}\right]{ }^{d} \omega_{0}^{N}
\end{aligned}
$$

in which eqs. (14) and (16) have been invoked and where ${ }_{0}^{N} \Omega^{d}=\left[0,\left({ }^{d} \omega_{0}^{N}\right)^{T}\right]^{T}$. In eq. (C.1) $F(\delta)$ is a $(3 \times 3)$ matrix defined by:

$$
F=\left[\begin{array}{ccc}
\delta_{s} & \delta_{v z} & -\delta_{v y} \\
-\delta_{v z} & \delta_{s} & \delta_{v x} \\
\delta_{v y} & -\delta_{v x} & \delta_{s}
\end{array}\right]
$$

\section{Part C2}

The second quaternion product expression on the right hand side of eq. (27) can be replaced by its equivalent matrix-column vector representation:

$$
\begin{aligned}
\delta \circ{ }_{0}^{N} \Omega^{a} & =\left[\begin{array}{cc}
\delta_{s} & -\delta_{v}^{T} \\
\delta_{v} & G
\end{array}\right]\left[\begin{array}{c}
0 \\
J_{r}(q) \dot{q}
\end{array}\right] \\
& =\left[\begin{array}{c}
-\delta_{v}^{T} \\
G
\end{array}\right] J_{r}(q) \dot{q}
\end{aligned}
$$

in which eqs. (14) and (15) have been invoked. In eq. (C.3) ${ }_{0}^{N} \Omega^{a}(q, \dot{q})$ has been expressed as a linear function of the generalized velocities by applying eqs. (3) and (4). The $(3 \times 3)$ matrix $G(\delta)$ in eq. (C.3) is defined by: 
40

$$
G=\left[\begin{array}{ccc}
\delta_{s} & -\delta_{v z} & \delta_{v y} \\
\delta_{v z} & \delta_{s} & -\delta_{v x} \\
-\delta_{v y} & \delta_{v x} & \delta_{s}
\end{array}\right] .
$$

It is easy to see that $G=-F+2 \delta_{s} I_{3 \times 3}$. 


\section{APPENDIX D \\ CLOSED LOOP TRANSLATIONAL ERROR SUBSYSTEM}

A closed loop error subsystem governing the translational motion of the end effector of a serial-link, kinematically redundant manipulator is derived in this Appendix. First consider the position tracking error $p_{e}$ of the end effector as defined by:

$$
p_{e}={ }^{d} p_{0}^{N}-{ }^{a} p_{0}^{N}
$$

where ${ }^{d} p_{0}^{N}$ and ${ }^{a} p_{0}^{N}(q)$ are defined below eq. (4). Since a velocity model is sought, consider the time derivative of eq. (D.1):

$$
\dot{p}_{e}={ }^{d} v_{0}^{N}-J_{t} \dot{q}
$$

in which eqs. (3) and (4) have been applied.

Our objective is to drive $\dot{p}_{e} \rightarrow 0_{3 \times 1}$. To accomplish this, a proportional and integral feedback control law is introduced:

$$
\dot{p}_{e}=-\left(K_{p} p_{e}+K_{i p} \int p_{e} d t\right)
$$

where $K_{p}$ and $K_{i p}$ are $(3 \times 3)$ positive definite gain matrices.

Eqs. (D.2) and (D.3) constitute a closed loop error system governing the translational motion of the end effector. 


\section{References}

[1] H. Goldstein, Classical Mechanics, 2nd Ed., Addison-Wesley, 1980, chap. 4, Appen $B$.

[2] T.R. Kane, P.W. Likens, and D.A. Levinson, Spacecraft Dynamics, McGraw-Hill, 1983, Chap. 1, Appen. I, II.

[3] E.T. Whittaker, A Treatise on the Analytical Dynamics of Particles and Rigid Bodies, 4th Ed, Cambridge University Press, 1988, chap. 1.

[4] J. Wittenburg, Dynamics of Systems of Rigid Bodies, B.G. Teubner Stuttgart, 1977, chap. 2.

[5] K.W. Spring, "Euler Parameters and the Use of Quaternion Algebra in the Manipulation of Finite Rotations: A Review," Mechanism and Machine Theory Vol. 21, No. 5, pp. 365-373, 1986.

[6] J.J. Craig, Introduction to Robotics, Mechanics, and Control, 2nd Ed., AddisonWesley, 1989.

[7] B.P. Ickes, "A New Method for Performing Digital Control System Attitude Computations using Quaternions," American Institute Aeronautics and Astronautics Journal, vol. 8, No. 1, pp. 13-17, Jan. 1970.

[8] J.S.-C. Yuan, "Closed Loop Manipulator Control Using Quaternion Feedback," IEEE Trans. Robotics and Automation vol. 4, no. 4, pp. 434-440, August 1988.

[9] S.-K. Lin, "Euler Parameters in Robot Cartesian Control," IEEE Conf. Robotics and Automation vol. 3, pp. 1676-1681, Philadelphia, PA, April 1988.

[10] J. Jansen, R. Kress, and S. Babcock, "Controller Design for a Force-Reflecting Teleoperator System with Kinematically Dissimilar Master and Slave," ASME J. Dynamic Systems, Measurement, and Control vol. 114, pp.641-649, December 1992.

[11] J.Y.S. Luh, M.W. Walker, and R.P.C. Paul, "Resolved-Acceleration Control of Mechanical Manipulators," IEEE Trans. Auton; atic Control vol. AC-25, No. 3, pp. 468-474, June 1980.

[12] D.N. Nenchev, "Redundancy Resolution through Local Optimization: A Review," J. of Robotic Systems Vol. 6, No. 6, pp. 769-798, December 1989.

[13] B. Siciliano, "Kinematic Control of Redundant Robot Manipulators: A Tutorial," J. of Intelligent and Robotic Systems Vol. 3, pp. 201-212, 1990. 
[14] J. Baillieul and D.P. Martin, "Resolution of Kinematic Redundancy" in Robotics, Proceedings of Symposia in Applied Mathematics, Vol. 41, pp.49-90, Louisville, Kentucky, January 1990 (American Mathematical Society Short Course Lecture Notes).

[15] P. Belmans and J-C. Culioli, "A New Approach to Solve the Kinematics Resolution of a Redundant Robot," Oak Ridge National Laboratory Technical Report ORNL/TM-11435 CESAR-89-53, March 1990.

[16] A.A. Maciejewski and C.A. Klein, "Obstacle Avoidance for Kinematically Redundant Manipulators in Dynamically Varying Environments," Inl'l J. Robotics Research Vol. 4, No. 3, pp 109-117, Fall 1985.

[17] R.G. Roberts and A.A. Maciejewski, "Near-Pseudoinverse Control of Kinematically Redundant Manipulators with the Constraint of Repeatability," 1991 IEEE Int'l Conf. Systems Engineering pp. 117-120, Dayton, OH, USA, August 1991.

[18] C.A. Klein and C.-H. Huang, "Review of Pseudoinverse Control for Use with Kinematically Redundant Manipulators," IEEE Trans. Systems, Man, and Cybernetics vol. smc-13, no. 3, pp. 245-250, March/April 1983.

[19] S. Hirose and S. Ma, "Redundancy Decomposition Control for Multi-Joint Manipulator," IEEE Int. Conf. Robotics and Automation vol. 1, pp. 119-124, Scottsdale, AZ, May 1989.

[20] J.M. Hollerbach and K.C. Suh, "Redundancy Resolution of Manipulators through Torque Optimization," IEEE J. Robotics and Automation vol. RA-3, no. 4, pp. 308-316, August 1987.

[21] R.V. Dubey, J.A. Euler, and S.M. Babcock, "Real-Time Implementation of an Optimization Scheme for Seven-Degree-of-Freedom Redundant Manipulators," IEEE Trans. Robotics and Automation Vol. 7, No. 5, pp. 579-588, October 1991.

[22] C.A. Klein and A.I. Chirco, "Dynamic Simulation of a Kinematically Redundant Manipulator System,” J. Robotic Systems Vol. 4, No. 1, pp 5-23, February 1987.

[23] R.H. Taylor, "Planning and Execution of Straight Line Manipulator Trajectories," IBM J. of Research and Development Vol. 23, pp 424-436, 1979.

[24] M.H. Ang and V.D. Tourassis, "Singularities of Euler and Roll-Pitch-Yaw Representations," IEEE Trans. Aerospace and Electronic Systems vol. AES-23, No. 3, pp. 317-324, May 1987. 
[25] D.E. Whitney, "The Mathematics of Coordinated Control of Prosthetic Arms and Manipulators," ASME J. Dynamic Systems, Measurement, and Control, vol. 122, pp. 303-309, Dec. 1972.

[26] K.S. Fu, R.C. Gonzalez, and C.S.G. Lee, Robotics: Control, Sensing, Vision, and Intelligence, McGraw-Hill, 1987, Appen. B.

[27] A.R. Klumpp, "Singularity-free Extraction of a Quaternion from a DirectionCosine Matrix," J. Spacecraft and Rockets vol. 13, no. 12, pp. 754-755, Dec. 1976.

[28] J.C.K. Chou, "Quaternion Kinematic and Dynamic Differential Equations," IEEE Trans. Robotics and Automation Vol. 8, No. 4, pp 53-64, February 1992.

[29] S.-K. Lin, "Coordinate Transformations with Euler Parameters as a Quaternion," IEEE Conf. Robotics and Automation vol. 1, pp. 33-38, Raleigh, NC, March-April 1987.

[30] R.A. Wehage, "Quaternions and Euler Parameters - A Brief Exposition," Computer Aided Analysis and Optimization of Mechanical System Dynamics Springer-Verlag Berlin Heidelberg, 1984, pp. 147-180.

[31] P.E. Nikravesh, "Spatial Kinematic and Dynamic Analysis With Euler Parameters," Computer Aided Analysis and Optimization of Mechanical System Dynamics Springer-Verlag Berlin Heidelberg, 1984, pp. 261-281.

[32] J.T.-Y. Wen and K. Kreutz-Delgado, "The Attitude Control Problem," IEEE Trans. Automatic Control vol. 36, No. 10, pp. 1148-1162, Oct. 1991.

[33] S.-K. Lin, "Singularity of a Nonlinear Feedback Control Scheme for Robots," IEEE Trans. on Systems, Man, and Cybernetics vol. 19, no. 1, pp. 134-139, Jan.-Feb. 1989.

[34] M.A. Unseren, "Input Relegation Control for Gross Motion of a Kinematically Redundant Manipulator," Oak Ridge National Laboratory Technical Report ORNL/TM-12165, October 1992.

[35] B. Noble and J.W. Daniel, Applied Linear Algebra, 2nd Ed., Prentice-Hall, 1977.

[36] F.G. Pin, M. Beckerman, P.F. Spelt, J.T. Robinson, and C.R. Weisbin, "Autonomous Mobile Robot Research Using the Hermies-III Robot," IEEE/RSJ Int'l Workshop on Intelligent Robots and Systems '89, September 4-6, 1989, Tsukuba, Japan, pp. 251-256. 
[37] C.R. Weisbin, B.L. Burks, J.R. Einstein, R.R. Feezell, W.W. Manges, and D.H. Thompson, "HERMIES-III: A Step Toward Autonomous Mobility, Manipulation and Perception," Robotica, Vol. 8, Part 1, January-March 1990, pp 7-12.

[38] J.N. Anderson and W.D. Combs, "Link Mass Properties of the CESAR Research Manipulator," Martin Marietta Energy Systems, Inc. Technical Report No. MCTR-0987-14, September 1987.

[39] M.A. Unseren and F.M. Hoffman, "Errata Report on Herbert Goldstein's Classical Mechanics, Second Edition," Oak Ridge National Laboratory Technical Report ORNL/TM-12176, January 1993. 


\section{INTERNAL DISTRIBUTION}

1. B. R. Appleton

2. J. E. Baker

3. A. L. Bangs

4. M. Beckerman

5. R. J. Carter

6. J. R. Einstein

7. C. W. Glover

8. F. M. Hoffman

9. J. F. Jansen

10. J. P. Jones

11. H. E. Knee

12. R. L. Kress

13-17. R. C. Mann

18. J. F. Manneschmidt

19. E. M. Oblow

20-24. F. G. Pin

25. V. Protopopescu
26. S. A. Raby

27. K. Rahmani

28. D. B. Reister

29. J. C. Schryver

30. P. F. Spelt

31. F. J. Sweeney

32. E. C. Uberbacher

33-37. M. A. Unseren

38. R. C. Ward

39-40. Laboratory Records Department

41. Laboratory Records, ORNL-RC

42. Document Reference Section

43. Central Research Library

44. ORNL Patent Section

\section{EXTERNAL DISTRIBUTION}

45. Dr. Peter Allen, Department of Computer Science, 450 Computer Science, Columbia University, New York, NY 10027

46. Dr. John S. Bay, Virginia Tech, Bradley Department of Electrical Engineering, 340 Whittemore Hall, Blacksburg, VA 24061-0111

47. Dr. Richard Becker, 2780 Oak Ridge Turnpike, Oak Ridge, TN 37830

48. Dr. Philippe F. R. Belmans, 19, rue Tiquetoune, 75002 Paris, FRANCE

49. Dr. Wayne Book, Department of Mechanical Engineering, J. S. Coon Building, Room 306, Georgia Institute of Technology, Atlanta, GA 30332

50. Professor Roger W. Brockett, Wang Professor of Electrical Engineering and Computer Science, Division of Applied Sciences, Harvard University, Cambridge, MA 02138

51. Professor John J. Dorning, Department of Nuclear Engineering and Physics, Thornton Hall, McCormick Rd., University of Virginia, Charlottesville, VA 22901

52. Dr. Stephen Dubowsky, Massachusetts Institute of Technology, Building 3, Room 469A, 77 Massachusetts Ave., Cambridge, MA 02139

53. Ross Giddings, Mechanical Engineering Department, University of Texas at Austin, 26 San Jacinto, Austin, TX 78712

54. Dr. Herbert Goldstein, 144-19 68th Rd., Flushing, NY 11367

55. Dr. H. Hemami, The Ohio State University, Department of Electrical Engineering, 205 Dreese Laboratory, 2015 Neil Ave,, Columbus, OH 43210-1272

56. Dr. Avi Kak, Department of Electrical Engineering, Purdue University, Northwestern Ave., Engineering Mall, Lafayette, IN 47907

57. Dr. James E. Leiss, Route 2, Box 142C, Broadway, VA 22815-9303

58. Dr. Oscar P. Manley, Division of Engineering, Mathematical, and Geosciences, Office of Basic Energy Sciences, ER-15, U.S. Department of Energy - Germantown, Washington, DC 20545 
59. Professor Neville Moray, Department of Mechanical and Industrial Engineering, University of Illinois, $1206 \mathrm{~W}$. Green St., Urbana, IL 61801

60. R. D. Rafler, 8906 Talbot Ave., Silver Spring, MD 20910

61. Dr. Bruno Siciliono, Dipartimento di Informatica e Sistemistica, Università degli Studi di Napoli Federico II, Via Claudio 21, 80125 Napoli, ITALY

62. Dr. Wes Snyder, Department of Radiology, Bowman Gray School of Medicine, $300 \mathrm{~S}$. Hawthorne Dr., Winston-Salem, NC 27103

63. Dr. Sabri Tosunoglu, Mechanical Engineering Department, University of Texas at Austin, 26 San Jacinto, Austin, TX 78712

64. Professor Mary F. Wheeler, Department of Mathematics, Rice University, P.O. Box 1892, Houston, TX 77251

65. Dr. Yong Zhou, 318 Norris Court, San Ramon, CA 94583

66. Office of Assistant Manager for Energy Research and Development, U.S. Department of Energy, Oak Ridge Operations Office, P.O. Box 2001, Oak Ridge, TN 37831-8600

67-68. Office of Scientific and Technical Information, P.O. Box 62, Oak Ridge, TN 37831 

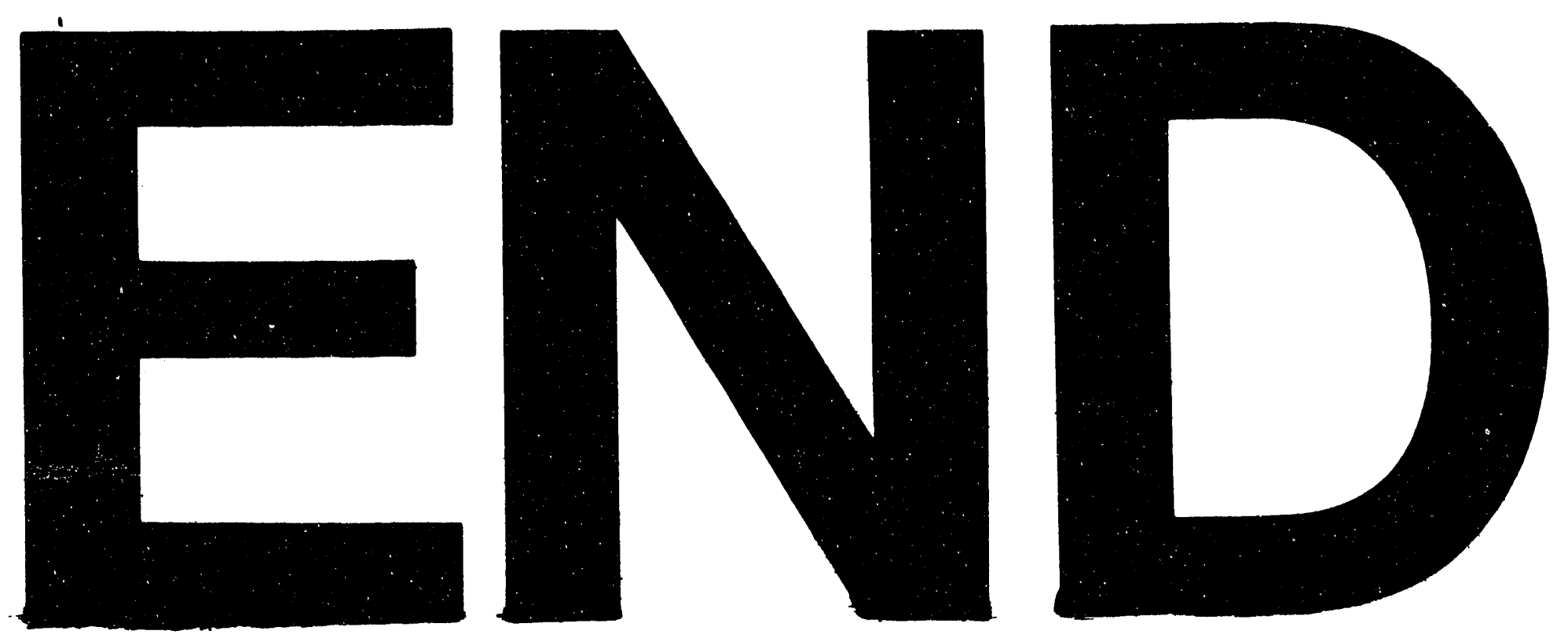

ES
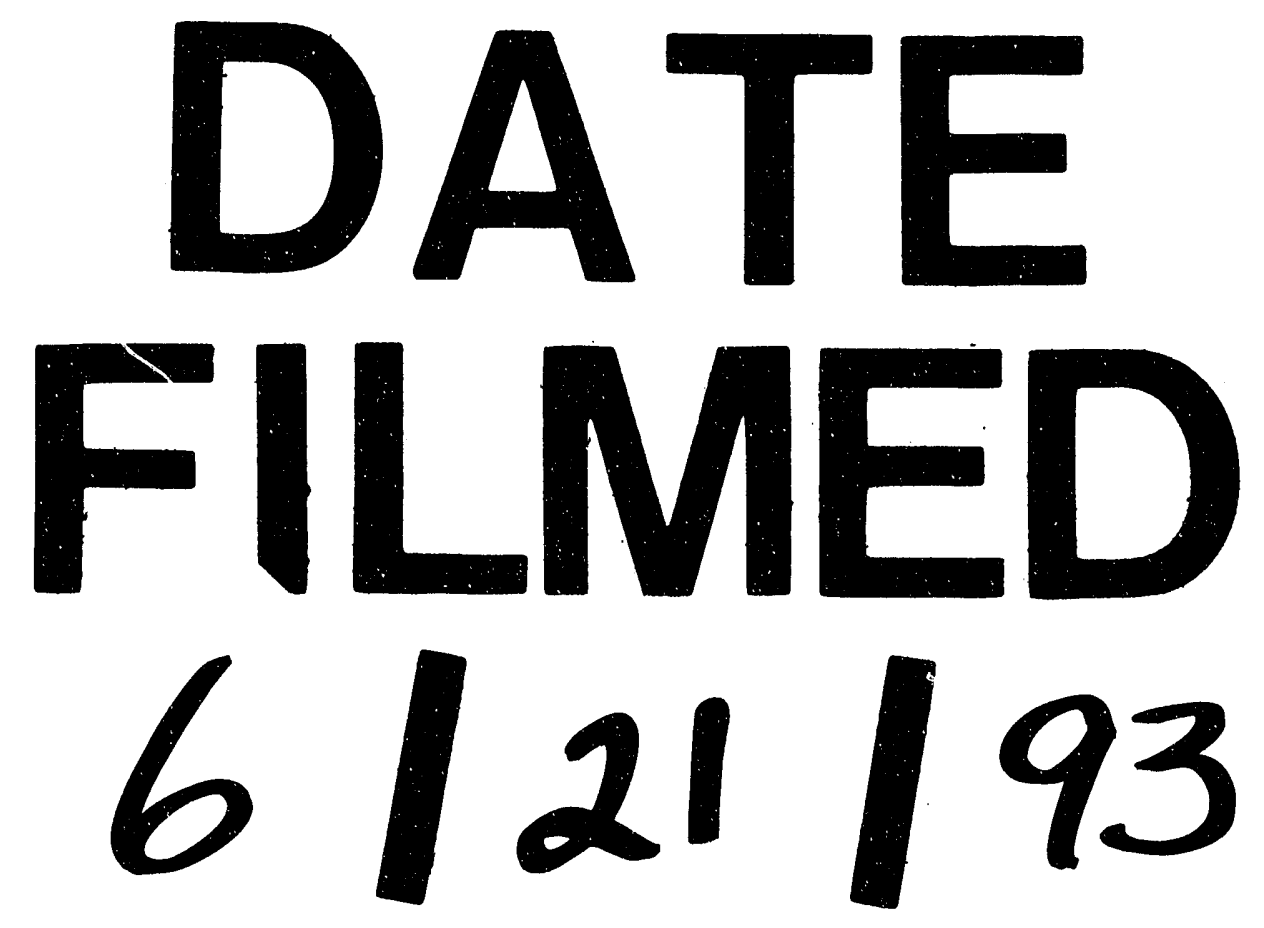
$\square$ 